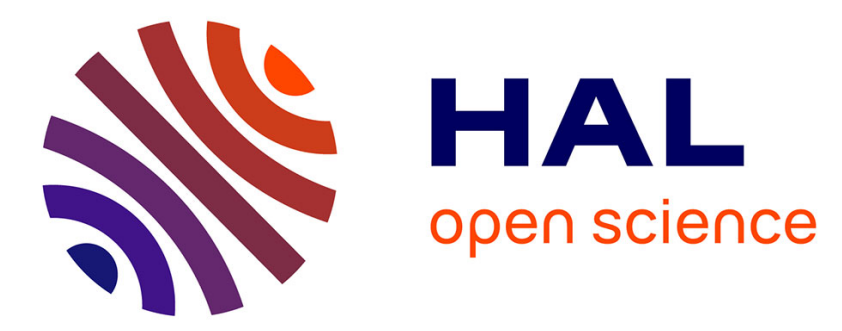

\title{
Dynamique des végétations sur le Causse du Larzac au cours des 8000 dernières années: contribution de l'étude anthracologique du Rajal del Gorp (Millau, Aveyron)
}

Léonor Liottier, Lucie Chabal, Matthieu Demierre

\section{- To cite this version:}

Léonor Liottier, Lucie Chabal, Matthieu Demierre. Dynamique des végétations sur le Causse du Larzac au cours des 8000 dernières années : contribution de l'étude anthracologique du Rajal del Gorp (Millau, Aveyron). Quaternaire, 2017, 28 (3), pp.337-352. 10.4000/quaternaire.8283 . hal-02311374

\author{
HAL Id: hal-02311374 \\ https://hal.science/hal-02311374
}

Submitted on 10 Oct 2019

HAL is a multi-disciplinary open access archive for the deposit and dissemination of scientific research documents, whether they are published or not. The documents may come from teaching and research institutions in France or abroad, or from public or private research centers.
L'archive ouverte pluridisciplinaire HAL, est destinée au dépôt et à la diffusion de documents scientifiques de niveau recherche, publiés ou non, émanant des établissements d'enseignement et de recherche français ou étrangers, des laboratoires publics ou privés. 


\title{
DYNAMIQUE DES VÉGÉTATIONS SUR LE CAUSSE DU LARZAC AU COURS DES 8000 DERNIÈRES ANNÉES : CONTRIBUTION DE L'ÉTUDE ANTHRACOLOGIQUE DU RAJAL DEL GORP (MILLAU, AVEYRON)
}

\author{
Léonor LIOTTIER ${ }^{1,2}$, Lucie CHABAL ${ }^{1}$, Matthieu DEMIERRE ${ }^{3}$
}

\section{RÉSUMÉ}

Le Rajal del Gorp est un aven karstique du Causse du Larzac ( $785 \mathrm{~m}$ alt). La grotte est fréquentée du Mésolithique (faciès Montclusien daté des $\mathrm{VII}^{\mathrm{e}}-\mathrm{VI}^{\mathrm{e}}$ millénaires B.C.) jusqu'à l'Antiquité tardive ( $\mathrm{IV}^{\mathrm{e}} \mathrm{s}$. A.D.). L'étude anthracologique s'organise en 16 strates successives qui en font la séquence la plus complète à ce jour pour cette région. Les résultats mettent en évidence une dynamique de végétation due au réchauffement climatique postglaciaire, avec dominance de pineraies de pin type sylvestre en début de séquence et un développement du chêne caducifolié au plus tard entre le Mésolithique moyen et le Néolithique ancien. Cette transition correspond à celle ce qui avait été décrite sur le Larzac, mais paraît plus tardive que sur le Causse Noir et que sur les sites méridionaux de plus basse altitude. La pinède se transforme progressivement en chênaie mixte (chêne caducifolié / pin), avec d'autres essences en très faible fréquence. La chênaie deviendra dominante dès la fin du Néolithique ancien et le pin (actuellement absent) persistera jusqu'à l'abandon du site. Les faibles taux de hêtre, identifié dès le Néolithique moyen / final et durant toute la séquence, marquent sa présence ancienne au sein d'une futaie de chêne mature. Cette dernière se transforme graduellement en taillis denses, abritant le hêtre qui reste cependant peu adapté au climat du Causse. Dès le Néolithique moyen / final, de rares taxons héliophiles liés à l'ouverture du milieu ou favorisés par le pâturage apparaissent et perdurent jusqu'à la fin de la séquence mais de façon très discrète, marquant une atteinte forestière modérée. Actuellement, les seules essences ligneuses qui persistent aux abords du site sont les buis et genévriers, épargnés par les troupeaux. Cette étude est une nouvelle synthèse paléoenvironnementale holocène du Causse du Larzac, à la lumière des résultats anthracologiques du Rajal del Gorp, qui apporte un éclairage inédit sur la végétation durant l'âge du Fer.

Mots-clés : Mésolithique, Néolithique, Protohistoire, Sud de la France, végétation, charbons de bois, paléoclimat, agro-pastoralisme

\section{ABSTRACT}

VEGETATION DYNAMICS ON THE CAUSSE DU LARZAC DURING THE LAST 8000 YEARS: NEW ANTHRACOLOGICAL RESULTS FROM THE SITE OF RAJAL DEL GORP (MILLAU, AVEYRON)

The archaeological site of Rajal del Gorp is located in asinkhole in the Causse du Larzac ( $785 \mathrm{~m}$ alt.). The cave was inhabited from the Mesolithic (earliest occupation dated to the VII - VI Millennia B.C.) up to the late Antiquity (IVth century A.D.).Charcoal analysis concerns a succession of 16 layers, constituting the most comprehensive sequence in this region. The vegetation dynamics outlined is clearly related with the post-glacial warming: dominance of Pinus type sylvestris in the early phase while deciduous oak expands at least from the Middle Mesolithic / Early Neolithic onwards. This transition occurs at the same time as that previously described in the Larzac, but is late when compared with the Causse Noir and other southern sites, at lower altitude. Pine forests are gradually transformed into mixed oak forests, where deciduous oaks and pines grow in sporadic association with other species. By the end of Early Neolithic, oaks become dominant, while pines - which are currently absent - remain present until the site is abandoned. The low frequencies of beech, identified as early as in the Middle / Late Neolithic and up to the Roman period, testify to the early presence of this species in the midst of mature oak forests. These forests which were gradually transformed into dense coppices, sheltered beech, which remained poorly adapted to the local climate. Scarce heliophilous taxa, associated with forest clearance or favoured by pastoral activities, appeared during the Middle / Late Neolithic period and persisted until the end of the sequence, but always with low frequencies. This is consistent with moderate anthropogenic impact. Nowadays, the only woody species still growing in the vicinity of the site are Buxus sempervirens and Juniperus communis which escaped grazing. This study proposes a palaeoenvironnemental synthesis and provides new data concerning the evolution of the Holocene woody vegetation in the Causse du Larzac. Those on the Iron Age are the first ever obtained in the region.

Keywords: Mesolithic, Neolithic, Protohistory, southern France, vegetation, wood charcoal, palaeoclimate, agro-pastoralism

\footnotetext{
${ }^{1}$ Institut des Sciences de l'Évolution, Université de Montpellier, CNRS, IRD, CIRAD, EPHE. CC 065 ; Place Eugène Bataillon, FBC*34095 MONTPELLIER Cedex 05, France.Courriel :leonor.liottier@umontpellier.fr, lucie.chabal@umontpellier.fr

${ }^{2}$ Université Paul Valéry, CNRS, Archéologie des Sociétés Méditerranéennes, UMR 5140 Université Paul Valéry, site de Saint-Charles, rue du Professeur Henri Serre, FR-34090 MONTPELLIER.

${ }^{3}$ Universités de Lausanne, ASA - Section d'Archéologie et des Sciences de l'Antiquité, Faculté des lettres, Bâtiment Anthropole, CH-1015 LAUSANNE. Courriel :matthieu.demierre@unil.ch
} 


\section{1 - INTRODUCTION}

Cet article présente l'étude anthracologique du site archéologique du Rajal Del Gorp (Millau, Aveyron), à la lumière des études paléoenvironnementales régionales passées. Ce site est implanté dans un aven karstique, au nord du Causse du Larzac (fig. 1), fréquenté entre le Mésolithique et l'Antiquité tardive ( $\mathrm{IV}^{\mathrm{e}} \mathrm{s}$. A.D.) de façon non continue (Demierre et al., 2015 ; Gruat et al., 2015). La grotte a fait l'objet de plusieurs campagnes de fouilles archéologiques entre 1978 et 1980 par A. Vernhet, puis entre 2008 et 2011 sous la direction de M. Demierre (Université de Lausanne). Les charbons de bois étudiés proviennent de ces dernières.

Le Causse du Larzac est caractérisé par un paysage fortement ouvert et marqué par les activités agropastorales anciennes, qui lui ont valu d'être inscrit au patrimoine mondial de l'UNESCO en 2011. Pourtant, l'histoire de la végétation du Larzac est restée méconnue pendant longtemps et continue à poser question. En effet, dès les années 1930, l'écologie passée des causses fait débat entre les écologues. Les premières hypothèses, selon lesquelles les causses étaient naturellement peu boisés (Flahault, 1901 ; Martonne (de), 1909) sont finalement abandonnées (Lio Tcheng Ngo, 1929 ; Prioton, 1932 ; Flahault, 1933, 1934 ; Marres, 1936). Dès lors, les pelouses steppiques du Larzac sont interprétées comme étant le résultat du déboisement. Mais quelles étaient alors les végétations sylvatiques présentes dans cette partie la plus méridionale des causses? Leur composition était-elle comparable aux lambeaux de forêt restés a priori intacts, à l'abri des interventions humaines, dans les canoles, failles naturelles qui incisent le plateau ? En quoi leur histoire estelle comparable ou diffère-t-elle de celle des causses et massifs voisins ? Et enfin, de quelle époque peut-on dater les débuts du déboisement et selon quels rythmes?

Ces questions ont suscité de vifs débats, notamment au moment où l'anthracologie a pris date dans le développement des sciences paléoenvironnementales, dès la fin des années 1960 par Vernet (1968). Les macrorestes végétaux, principalement des charbons de bois, constituent en effet la majorité des sources d'étude sur le Larzac, avec les études des grottes de Sargel (Néolithique et Chalcolithique), des Cascades (Néolithique et âge du Bronze moyen) et des Treilles (Néolithique final) (Vernet, 1968, 1972, 1973), ainsi que l'abri des Usclades (de l'Épipaléolithique au Néolithique ancien) et celui des Canalettes (Moustérien, autour de $73500 \pm 6000$ TL B.P.) (Valladas $e t$ al., 1987 ; Théry et al., 1996 ; Théry-Parisot, 1998 ; ThéryParisot \& Meignen, 2000). Le site de la Poujade (KraussMarguet, 1981), situé un peu plus au nord, de l'autre côté de la Dourbie, constitue également une séquence anthracologique de référence, mais sur le Causse Noir.

Les empreintes foliaires des travertins des gorges du Tarn donnent des points de comparaison (Vernet, 1985).

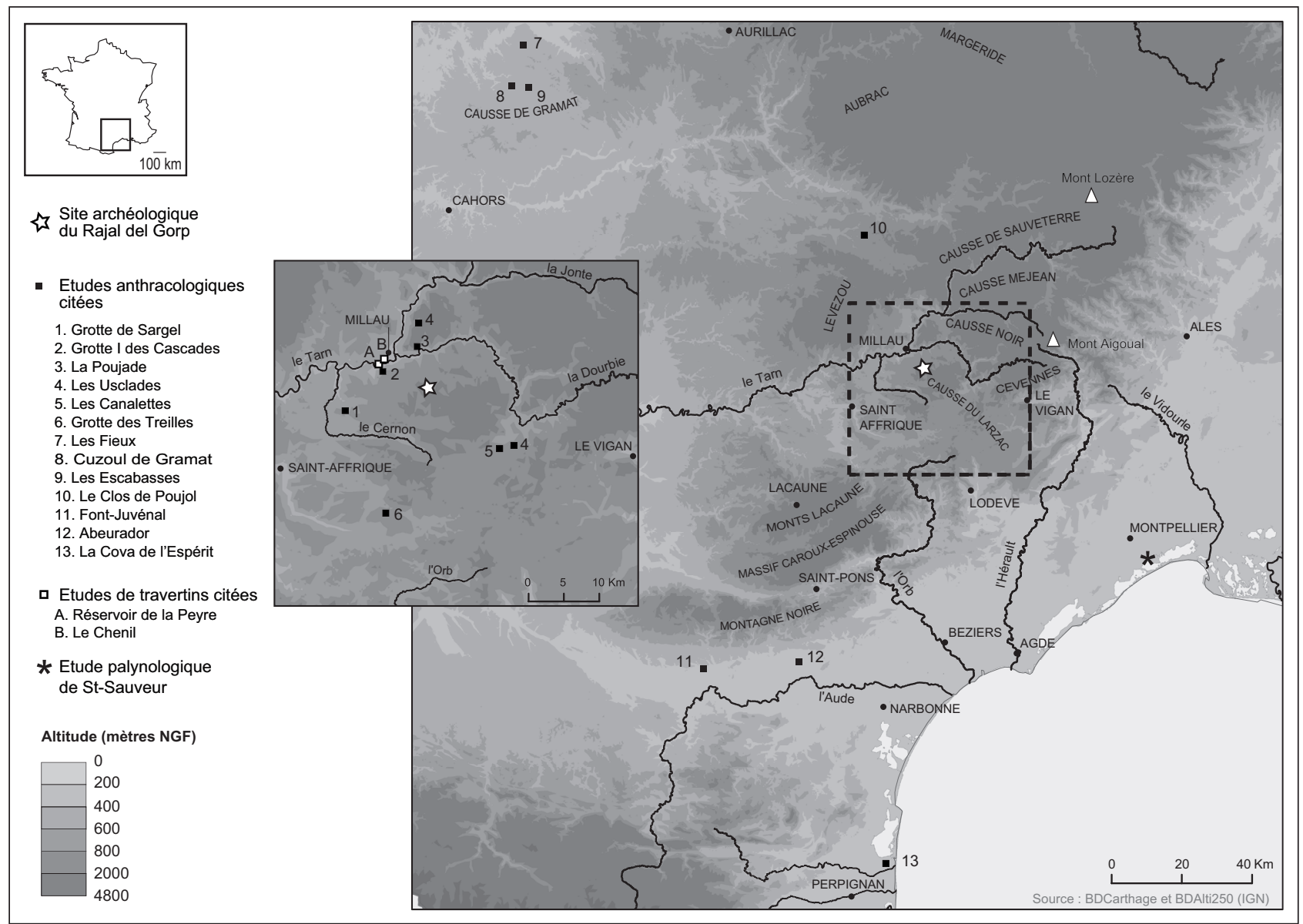

Fig. 1 : Carte de localisation des principaux sites mentionnés dans le texte. Point noir: ville; triangle: sommet; ligne: rivière.

Fig. 1: Location map of the main sites cited in the text. Black point: city; Triangle: mountain peak; Line: river; Star: Rajal del Gorp location; Black square: anthracological studies; White square: Travertines studies; Black star: Palynological sites; Grey scale: elevation (meters a.s.l.). 
Les études palynologiques sont quasi inexistantes sur les causses du fait du substrat calcaire et de la dynamique des écoulements, qui ne permettent pas les conditions d'humidité et d'acidité propres à la conservation du pollen. Les études palynologiques les plus proches proviennent des tourbières localisées sur les monts alentours de l'Aubrac, du Lévézou, de l'Aigoual, de Lacaune, du massif de l'Espinouse et de la Montagne Noire. Les travaux fondateurs de J.-L. de Beaulieu, et ses collaborations avec A. Pons et M. Reille (Beaulieu (de) 1981 ; Beaulieu (de) et al.1982, 1984, 1985) servent de référence quant aux connaissances des dynamiques de végétation passée dans la partie méridionale du Massif central. Les études de palynologie plus récentes dans ces secteurs ont également permis d'affiner la datation, mais aussi la perception de ces variations, dépendantes de facteurs climatiques et anthropiques (Pulido Avila, 2006 ; Bottollier-Curtet \& Muller, 2008 ; Faure, 2012).

L'étude anthracologique du Rajal del Gorp permet de proposer un nouvel éclairage sur l'évolution des boisements depuis le réchauffement postglaciaire jusqu'à l'Antiquité.

\section{2 - CADRES ENVIRONNEMENTAL, ARCHÉOLOGIQUE ET CHRONOLOGIQUE DE L'ÉTUDE}

\section{1 - SITUATION DU SITE}

L'aven du Rajal del Gorp se trouve sur le Causse du Larzac, à 785 m d'altitude, au pied d'un chaos ruiniforme qui lui a transmis son nom. L'Aven résulte de l'érosion mécanique et chimique de bancs calcaires fissurés, qui forment ainsi de véritables réseaux karstiques. L'eau est très rare en surface, mais présente aux abords du site sous forme de cours d'eau temporaires lors des fortes pluies. Ailleurs, le plateau est entaillé de vallées creusées par les rivières et ruisseaux, alimentés par les eaux souterraines du plateau. Les vallées de la Dourbie et du Tarn, en limite nord du plateau, sont les plus proches du site. Le substratum, du Jurassique moyen et supérieur, est essentiellement constitué de calcaires et de dolomies souvent affleurants. Les sols sont minces et parfois très superficiels, pierreux, secs et érodés (IGN, 2016).

\section{2 - CLIMAT ET VÉGÉTATION ACTUELS}

Le site se trouve aujourd'hui dans l'étage bioclimatique supraméditerranéen, à la base de l'étage montagnard, caractérisé par la série du chêne caducifolié, sous influences climatiques méditerranéenne et montagnarde. Les pineraies de pin sylvestre forment la série mixte du chêne pubescent / sessile et du pin sylvestre (Vernet, 1968, 1985 ; Barbero et al., 1971, 1978).

Le Causse du Larzac offre un paysage contrasté (fig. 2). Au nord du plateau où se trouve le site, on n'observe plus que des bosquets de chênes, très amoindris par l'activité humaine, et des étendues dévolues au buis et au genévrier cade, épargnés par les troupeaux, voire uniquement des zones herbacées. Les pineraies bien constituées du nordest du plateau, ainsi que de vieux individus au sud, sont

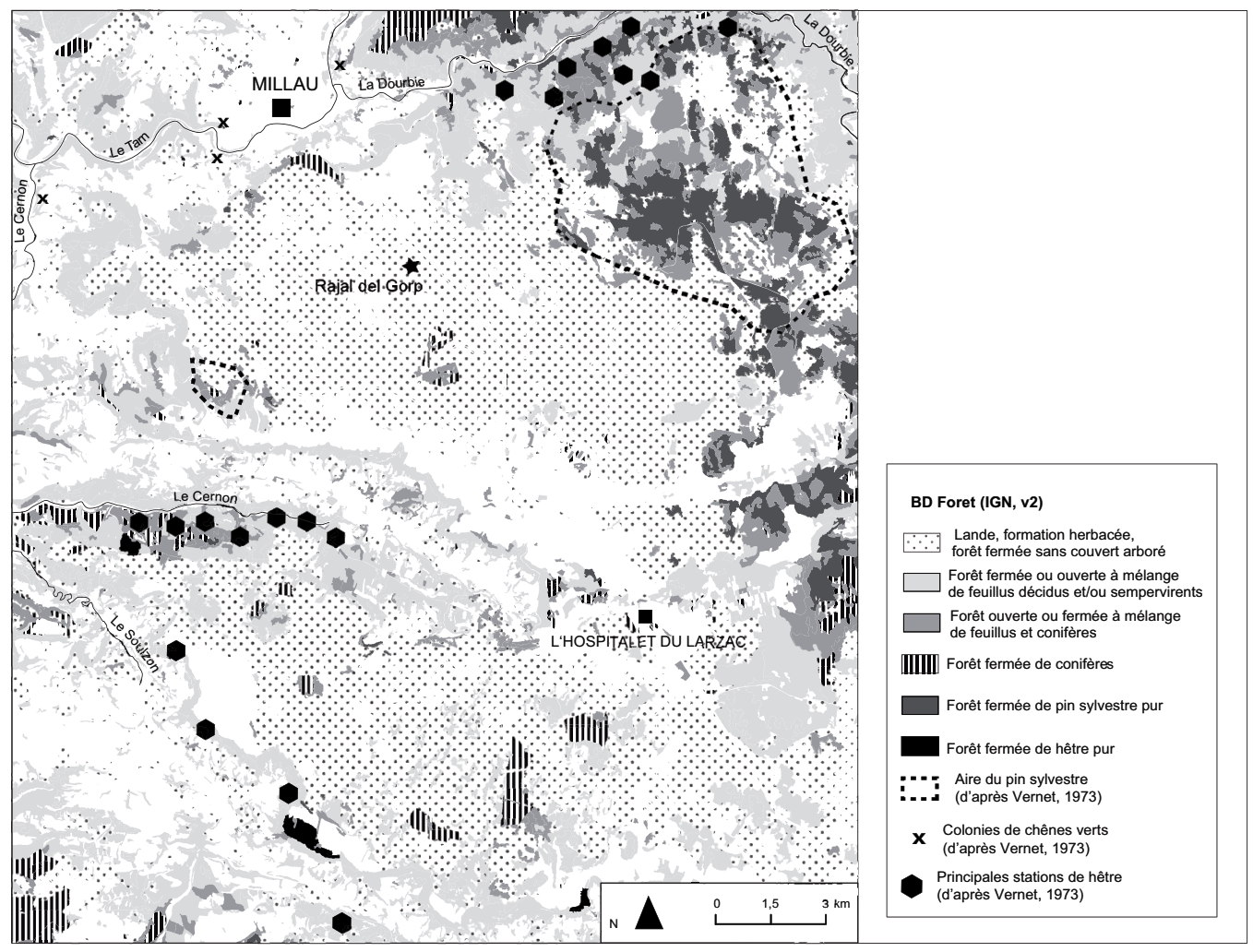

Fig. 2 : Carte de la végétation du Causse du Larzac (d'après Vernet, 1972 et l'IGN 2016$).$

Fig. 2: Vegetation types on Causse du Larzac (after Vernet, 1972 and IGN datas, 2016). Vertical stripes: Closed or cleared deciduous and/or evergreen forest; Horizontal stripes: Closed conifer forest; Black: closed pure beech forest; Dark grey: Closed pure Scots Pine forest; Medium grey: Closed or open mixed hardwood and conifer forest; Light grey: Open pure hardwood forest; Spotted areas: Moorland, herbaceous formation, closed forest without tree cover; Polygons: Main beech areas (after Vernet, 1973); Dotted lines area: Area of the Scots Pine (after Vernet, 1973). 
de faible étendue (Vernet, 1972a). Le pin sylvestre est très peu représenté actuellement sur le causse, partout où le démantèlement de la forêt et l'érosion des sols ne sont plus favorables à sa régénération. Quelques parcelles plantées de pin noir sont présentes dans le centre du plateau, vestiges des campagnes de reboisement des causses des années 1960 (Boulant \& Lepart, 2008). Les vallons, principalement en marges du plateau, abritent des peuplements de feuillus en mélange, dont le chêne pubescent (Quercus pubescens), le chêne vert (Quercus ilex), le hêtre (Fagus sylvatica), ou le noisetier (Corylus avellana). Le hêtre ne se maintient qu'aux altitudes les plus élevées du causse, à la faveur des nébulosités atmosphériques, et dans des vallons sur les versants en ubac (voir 5.5.2).

\section{3 - STRATIGRAPHIE ET FONCTIONS DU SITE}

La stratigraphie du site se compose d'une succession de 10 niveaux échelonnés entre -17 et $-27 \mathrm{~m}$ d'altitude par rapport à la surface. Ils se situent majoritairement dans la seconde cavité, qui s'étend sur une longueur de $25 \mathrm{~m}$, pour une largeur comprise entre 7 et $15 \mathrm{~m}$ (Demierre et al., 2015) (fig. 3).

Le manque de visibilité dans la grotte et les conditions de fouilles difficiles ont conduit à un prélèvement et à un tamisage quasi exhaustifs du sédiment, par Unités de Fouilles (UF). Ainsi toutes les périodes représentées ont pu être traitées en anthracologie.

L'étude du mobilier archéologique a révélé plusieurs fonctions plus ou moins bien cernées de la cavité depuis le Mésolithique (Montclusien), jusqu'à la période romaine (Bas Empire). Les premières fréquentations préhistoriques, datées du Mésolithique moyen par le matériel archéologique, étaient probablement temporaires, saisonnières, principalement liées à la pratique de la chasse. La datation radiocarbone du niveau 44 , inédite à ce jour, permet de dater ces niveaux autour de 8247 - 7937 cal. B.C. $(8900 \pm 50$ B.P., Poz-77389). Le niveau 43 sus-jacent ne s'en différencie guère sur le plan de l'anthracologie, mais contient du matériel archéologique associé au Mésolithique final ou au Néolithique ancien (Demierre \& Mendiburu, 2011) (fig. 4). Le hiatus avec les niveaux du Néolithique ancien, difficile à estimer du fait de l'imprécision chronologique de l'UF 43, pourrait donc avoir duré entre quelques centaines d'années et un peu plus de 2000 ans.

Entre le Néolithique ancien et la période laténienne (fig. 4 et 5), la cavité aurait servi de halte, plus que d'occupation (dans le cadre d'activités agropastorales ou de chasse), voire de grotte sépulcrale (présence de céramiques fines du Bronze final, caractéristiques de la

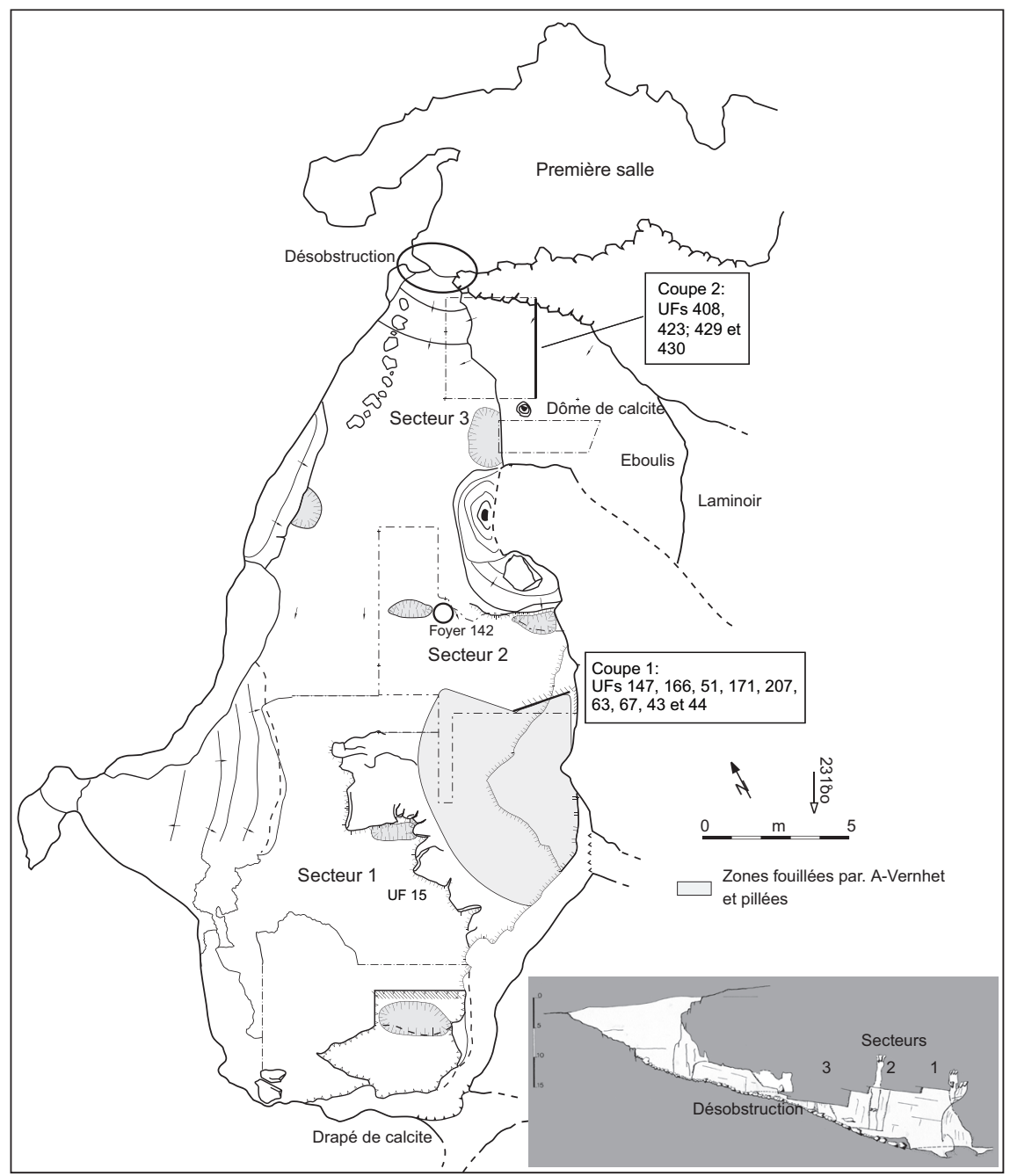

Fig. 3 : Plan de la grotte du Rajal del Gorp avec la localisation des coupes $\mathbf{1}$ et 2.

Fig. 3: Plan of the Rajal del Gorp cave showing location of stratigraphic cross section 1 and 2. 


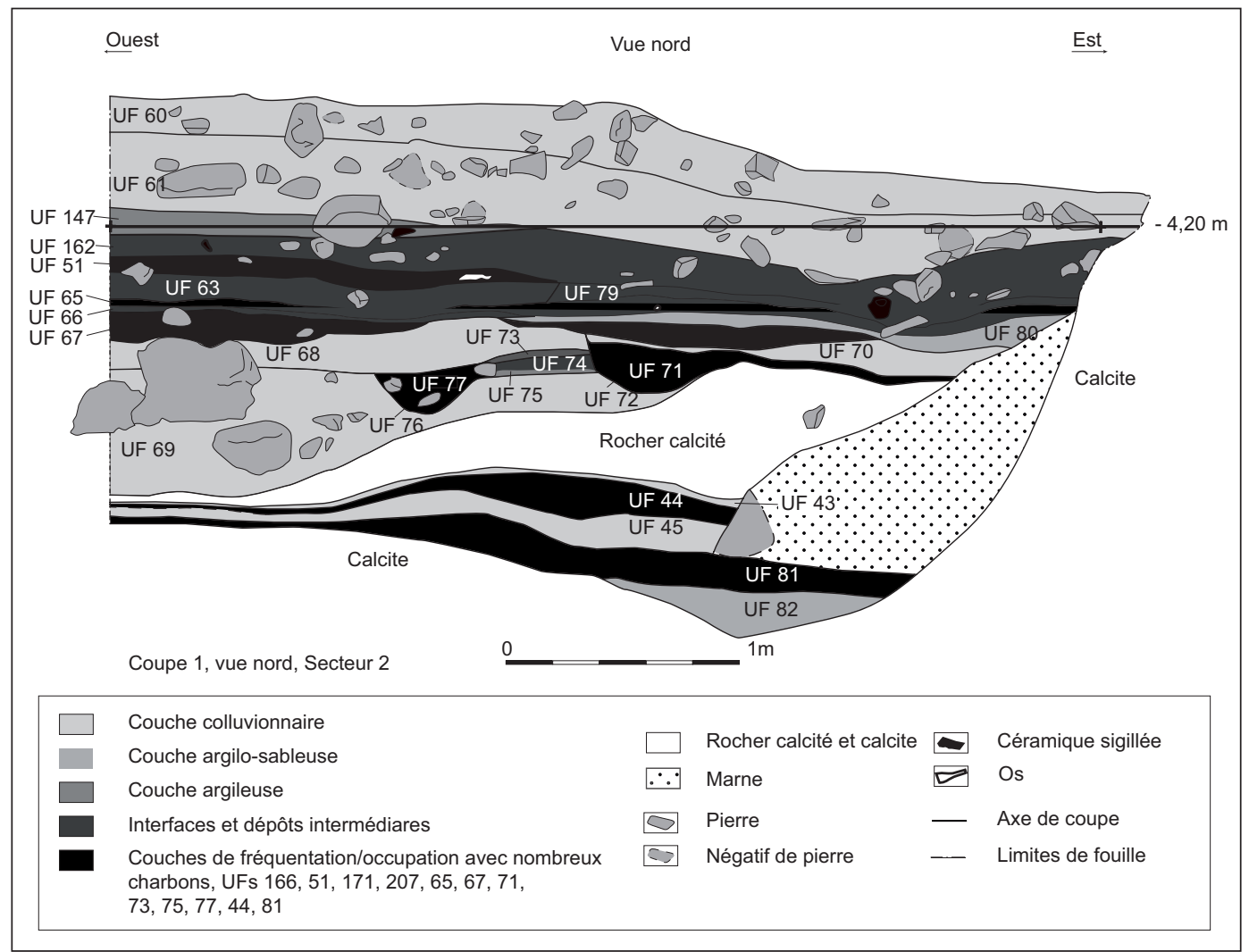

Fig. 4 : Coupes 1, vue nord, secteur 2 du Rajal del Gorp.

Fig. 4: Cross section 1, from north view, zone 2 of the Rajal del Gorp.

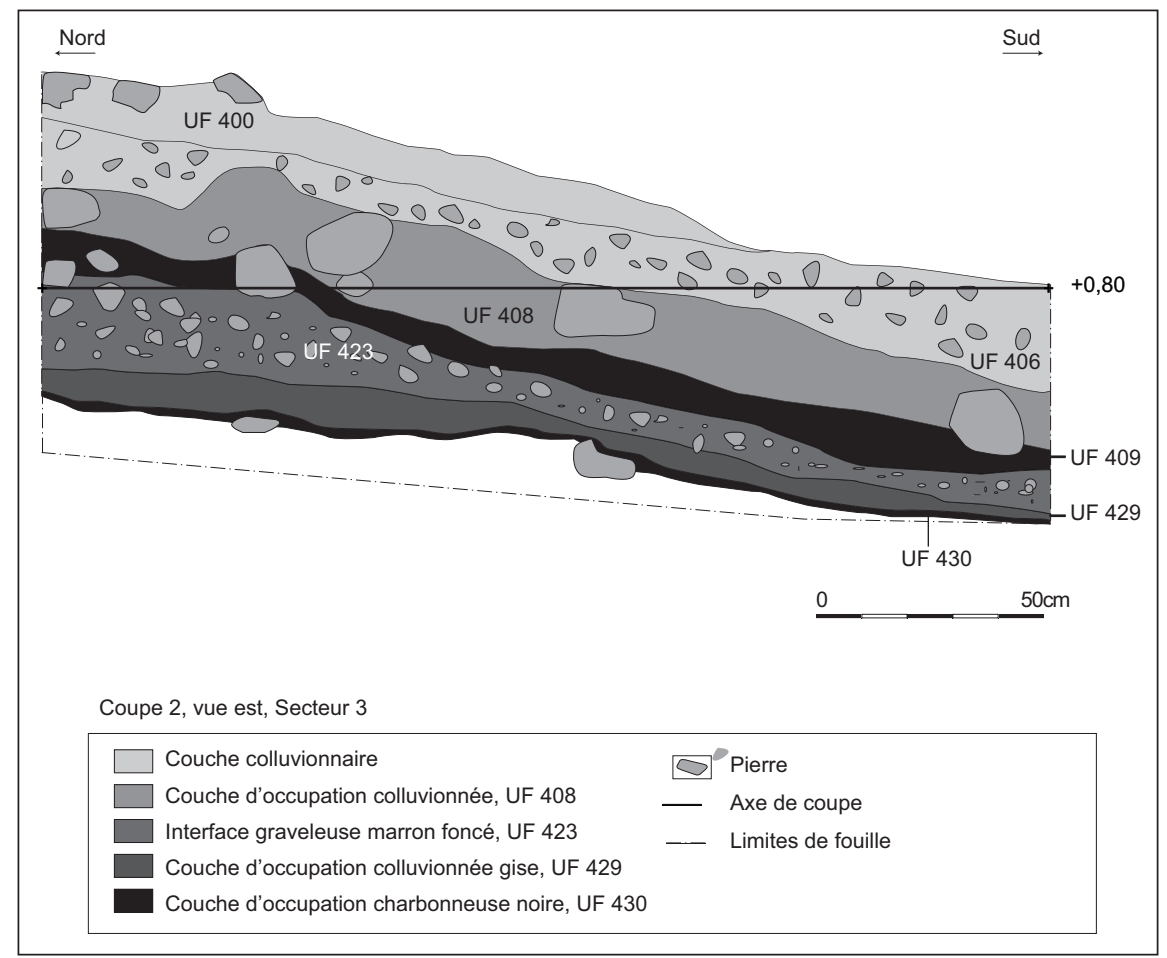

Fig. 5 : Coupes 2, vue nord, secteur 2 du Rajal del Gorp.

Fig. 5: Cross section 1, from east view, zone 3 of the Rajal del Gorp.

sphère funéraire et d'os épars de sept individus). Les UF 403 et 429 sont attribuées au Néolithique moyen / final (fig. 4 et 5), bien qu'elles contiennent des intrusions plus récentes. Les UF 423 et 63 sont des UF d'interface comportant du matériel visiblement remanié, situées entre le Néolithique final et le premier âge du Fer. En effet, l'étude archéozoologique a mis en évidence de possibles perturbations et intrusions de matériel plus récent dans ces niveaux, l'UF 429 ayant révélé des restes de poule domestiquée (cet animal n'étant pas domes- 
tiqué avant l'âge du Bronze / Fer). Quoi qu'il en soit, les incertitudes chronologiques au sein de cette période ne compromettent pas la perception d'une évolution forestière régulière.

Après une période d'abandon, la grotte est à nouveau fréquentée comme sanctuaire à partir de la période laténienne, à la fin du $\mathrm{II}^{\mathrm{e}} \mathrm{s}$. B.C. jusqu'à son abandon $\mathrm{au} \mathrm{IV} \mathrm{I}^{\mathrm{e}} \mathrm{S}$. A.D. De nombreux dépôts de céramiques, parures et monnaies, mais aussi d'offrandes animales et végétales caractérisent ces niveaux.

Le Rajal del Gorp, en dépit de hiatus probables, ou tout au moins d'incertitudes chronologiques, a donné la séquence anthracologique la plus complète à ce jour pour le Causse du Larzac, en termes d'évolution du couvert forestier et d'intensification de l'utilisation de cette ressource, recouvrant les périodes clés des débuts du Néolithique et de la période romaine.

\section{4 - DATATIONS ET CHRONOLOGIES}

Cette étude fait appel à des datations et chronologies, du Rajal del Gorp ou extérieures au site, issues de sources différentes et parfois anciennes. Concernant les dynamiques de végétation des Grands Causses, nous nous basons principalement sur les études et synthèses de Vernet $(1972,1968,1985)$ et Krauss-Marguet (1980, 1981) dont les datations C14 de référence étaient exprimées en dates B.P. ou B.C. non calibrées. Les dates de chacun des sites et niveaux ayant servi à construire le phasage de référence, ont été calibrées grâce au logiciel Oxcal 4.3, utilisant la courbe de calibration IntCal 13 (Bronk Ramsey, 2009). Il en est de même pour toutes les dates exprimées en cal. B.C. évoquées, regroupées dans la figure 6. Les intervalles de datation sont exprimés avec une probabilité de 95,4\%. La datation par thermoluminescence des Canalettes est exprimée en TL B.P. (Valladas

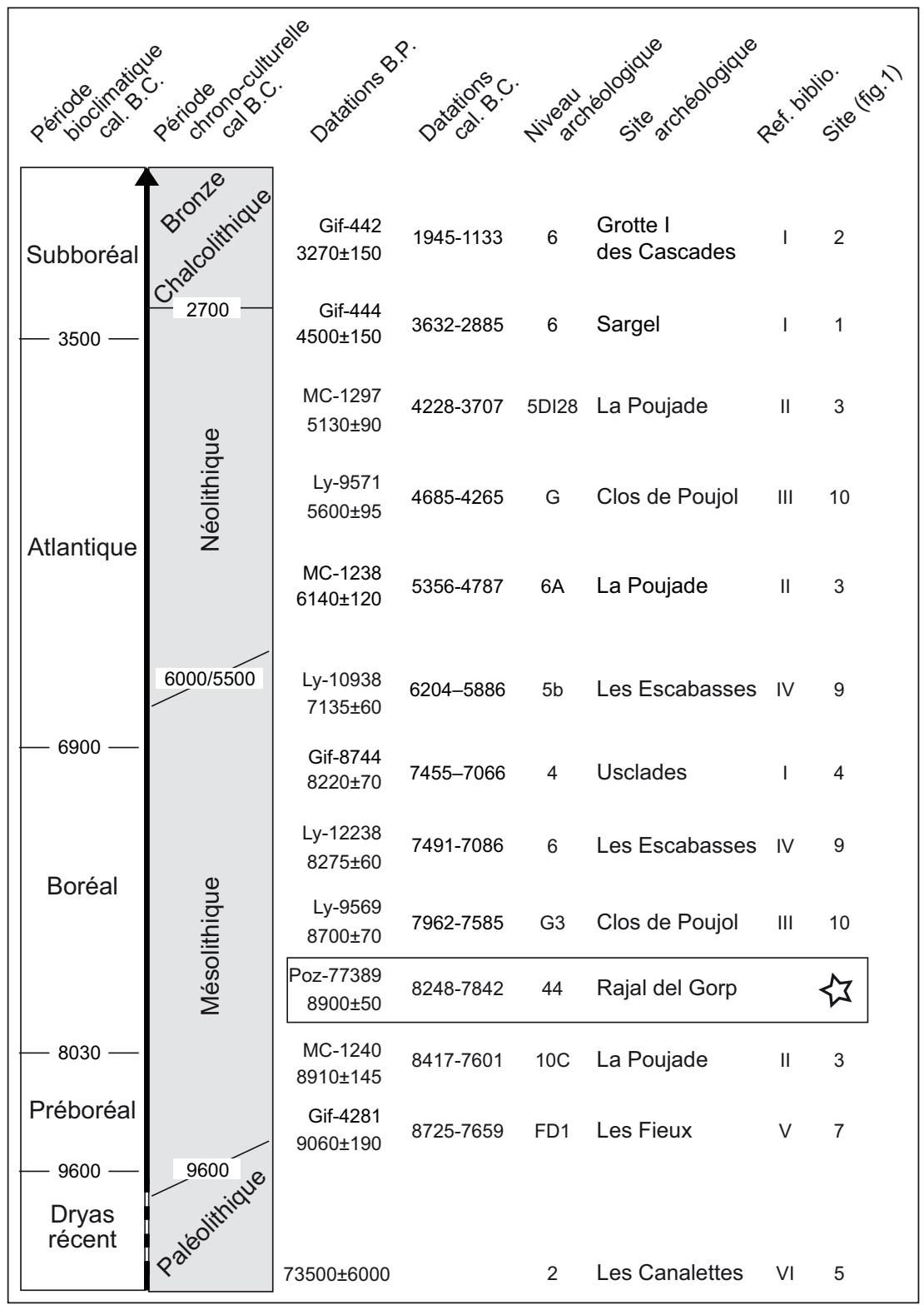

Fig. 6 : Périodes et datations des niveaux des principaux sites mentionnées dans le texte.

D’après : I. Delibrias et al., 1970 ; Costantini, 1984. II. Krauss-Marguet, 1981. III. Henry, 2011. IV. Valdeyron et al., 2008,2011 ; Henry et al., 2013. V. Champagne et al., 1990 ; Henry et al., 2013. VI. Valladas et al., 1987 ; Théry et al., 1996.

Fig. 6: Periods and datations of the main levels sites cited in the text (from: I. Delibrias et al., 1970; Costantini, 1984. II. Krauss-Marguet, 1981. III. Henry, 2011. IV. Valdeyron et al., 2008, 2011 ; Henry et al., 2013. V. Champagne et al., 1990 ; Henry et al., 2013. VI. Valladas et al., 1987 ; Théry et al., 1996). 
et al, 1987). Les datations B.P. issues des synthèses sur la diffusion postglaciaire du chêne décidu (Brewer et al., 2002 ; Petit et al., 2002) ne sont pas calibrées.

\section{3 - MATÉRIEL ET MÉTHODE}

Les charbons de bois étudiés proviennent de la sédimentation des sols de fréquentation, ainsi que de structures archéologiques, telles qu'un foyer et un trou de poteau. Ces charbons de bois sont les restes du combustible utilisé à des fins domestiques ou cultuelles dans la grotte. En dépit d'usages du bois variables, mais du fait de fréquentations de courte durée sur le site, le bois utilisé devait provenir de l'environnement proche, ce qui explique une perception anthracologique régulière.

Le prélèvement total des sédiments des UF, et leur tamisage à l'eau et/ou par flottation aux mailles 5 et $2 \mathrm{~mm}$ pour la plupart des échantillons (Demierre et al., 2015), ont garanti la récupération optimale des charbons de bois pour l'étude de la composition et de la structure de la végétation, conformément aux méthodes actuelles de la discipline (Vernet, 1968 ; Chabal 1997 ; Chabal et al., 1999 ; Théry-Parisot, 2001). Un total de 16 UF (Unités de Fouille) a été étudié, permettant l'identification de 1888 charbons de bois (tab. 1).

La méthode d'identification consiste en l'observation, au microscope photonique à réflexion (grossissements 50 et à 1000 fois), des trois plans anatomiques du bois par cassures fraîches des charbons de bois. Les caractéristiques anatomiques du bois, conservées malgré la carbonisation, sont comparées à celles décrites dans les atlas de référence (Schweingruber, 1990 ; Vernet et al., 2001), et aux charbons de bois de la collection de comparaison de l'ISEM. La bonne conservation des charbons de bois a permis le plus souvent leur détermination au genre ou à l'espèce. L'hésitation entre deux espèces ou genres justifie parfois la création d'un « taxon-valise ». C'est le cas d'Acer campestre et Acer monspessulanum, identifiables à l'espèce selon les critères suivants : les vaisseaux, en plan transversal, ont un diamètre moyen de 20 à $50 \mu \mathrm{m}$ chez Acer monspessulanum, de 50 à $100 \mu \mathrm{m}$ chez $A$. campestre et la largeur des rayons observables en plan tangentiel peut atteindre 8 cellules pour $A$. monspessulanum, contre 5 cellules au maximum pour $A$. campestre (Jacquiot et al., 1973 ; Schweingruber, 1990). Mais, dans de nombreux cas, les fragments présentaient des caractéristiques intermédiaires. L'identification est alors notée Acer campestre/monspessulanum, conformément aux conventions européennes (base de données ArboDat). Une telle incertitude peut-être due : soit à une observation contrainte par la petite taille des charbons de bois, soit à des individus possédant une variation d'anatomie à cause de paramètres stationnels (par exemple, l'humidité atmosphérique ou du sol peut influencer la taille des pores), soit encore à la présence d'individus hybrides. Par ailleurs, les fragments sont identifiés au genre Acer,

\begin{tabular}{|c|c|c|c|c|c|c|c|c|c|c|c|c|c|c|c|c|c|}
\hline Périodes & \multicolumn{2}{|c|}{ Mésolithique } & \multicolumn{3}{|c|}{ Néolithique ancien } & \multicolumn{2}{|c|}{$\begin{array}{l}\text { Néolithique } \\
\text { moyen/final }\end{array}$} & \multicolumn{2}{|c|}{$\begin{array}{c}\begin{array}{c}\text { Néolithique final à } \\
\text { Fer I }\end{array} \\
\text { a }\end{array}$} & \multicolumn{4}{|c|}{ La Tène finale } & \multicolumn{3}{|c|}{ Haut-Empire } & \multirow[t]{2}{*}{$\begin{array}{l}\text { Bas- } \\
\text { Empire }\end{array}$} \\
\hline Datation $14 \mathrm{C}$ (cal B.C.) & $\begin{array}{l}8248- \\
7842 \\
\end{array}$ & & & & & & & & & & & & & & & & \\
\hline $\begin{array}{c}\text { UF } \\
\text { TAXONS }\end{array}$ & 44 & 43 & 67 & 142 & 245 & 403 & 429 & 423 & 63 & 207 & 171 & 51 & 166 & 162 & 147 & 408 & 15 \\
\hline $\begin{array}{l}\text { Acer campestre } \\
\text { Erable champêtre }\end{array}$ & & & & & & & & & 1 & & & & & & & & \\
\hline $\begin{array}{l}\text { Acer monspessulanum } \\
\text { Erable de Montpellier }\end{array}$ & 2 & 3 & 1 & & & & & & & & & & & 12 & 1 & & 1 \\
\hline $\begin{array}{l}\text { Acer monspessulanum/campestre } \\
\text { Erable de Montpellier/champêtre }\end{array}$ & & & 1 & & & & & & & & & & & & 3 & 1 & 1 \\
\hline $\begin{array}{l}\text { Acer } \\
\text { Erable }\end{array}$ & & 1 & & & & & & & 1 & & & & & 4 & & & \\
\hline $\begin{array}{l}\text { Alnus } \\
\text { Aulne }\end{array}$ & & & & & & 1 & & & & & & & & & & & \\
\hline $\begin{array}{c}\text { Buxus sempervirens } \\
\text { Buis }\end{array}$ & & & & & & 24 & & 6 & & 1 & & 2 & & & 1 & 3 & 18 \\
\hline $\begin{array}{c}\text { Corylus avellana } \\
\text { Noisetier }\end{array}$ & & & & & 1 & & & & & 2 & & & 1 & & 3 & & 2 \\
\hline $\begin{array}{c}\text { Fagus sylvatica } \\
\text { Hêtre commun }\end{array}$ & & & & & & 1 & 1 & 1 & 1 & 5 & & & & & & 1 & 7 \\
\hline $\begin{array}{l}\text { Ligustrum vulgare } \\
\text { Troène commun }\end{array}$ & & & & & 1 & & & & & & & 1 & & & & & \\
\hline $\begin{array}{l}\text { Pinus type sylvestris } \\
\text { Pin de type sylvestre }\end{array}$ & 89 & 88 & 64 & 52 & 32 & 5 & 43 & 23 & 30 & 29 & 23 & 11 & 5 & 17 & 8 & 9 & 41 \\
\hline $\begin{array}{l}\text { Populus } \\
\text { Peuplier }\end{array}$ & 1 & & & & & & & & & & & & & & & & \\
\hline $\begin{array}{c}\text { Prunus cf. domestica/spinosa } \\
\text { Prunier/prunellier }\end{array}$ & & & & & & & & & & & & & & & & & 1 \\
\hline $\begin{array}{l}\text { Quercus deciduous } \\
\text { Chêne décidu }\end{array}$ & 1 & 1 & 32 & 24 & 57 & 61 & 54 & 69 & 56 & 59 & 76 & 82 & 93 & 61 & 76 & 83 & 115 \\
\hline $\begin{array}{l}\text { Quercus } \\
\text { Chêne }\end{array}$ & & & & & & & 1 & & & & & & & & & & \\
\hline $\begin{array}{l}\text { Rosaceae Maloideae } \\
\text { Rosacée Maloïdée }\end{array}$ & 1 & & & & & 1 & & & & 1 & & 1 & & & & 1 & 4 \\
\hline $\begin{array}{l}\text { Rosaceae Maloideae cf. Crataegus } \\
\text { Cf. aubépine }\end{array}$ & 1 & 1 & & & 1 & & 1 & & & & & & & & & & 1 \\
\hline $\begin{array}{l}\text { Rosaceae Prunoideae } \\
\text { Rosacée Prunoïdée }\end{array}$ & & & & & & & & & & 1 & & & & & & & \\
\hline $\begin{array}{l}\text { Rosaceaee Prunoideae 4-5s. } \\
\text { Rosacée Prunoïdée 4-5s. }\end{array}$ & & & & & & & & & & 1 & & & & & & & \\
\hline $\begin{array}{l}\text { Rosaceae Prunoideae 6-8s. } \\
\text { Rosacée Prunoïdée 6-8s. }\end{array}$ & & & & & & & & & & 1 & & & & & & & \\
\hline $\begin{array}{l}\text { Salix } \\
\text { Saule }\end{array}$ & 2 & & & & & & & & & & & & & & & & 2 \\
\hline $\begin{array}{c}\text { Ulmus } \\
\text { Orme }\end{array}$ & & & & & & & & & & & & 1 & & & & & \\
\hline Indéterminé & 2 & 2 & & & 1 & & & & & & & 1 & & & & 1 & 1 \\
\hline Indéterminable & 2 & 4 & 2 & 24 & 7 & 2 & & 1 & 11 & & 1 & 1 & 1 & 6 & 8 & 1 & 6 \\
\hline Total & 101 & 100 & 100 & 100 & 100 & 95 & 100 & 100 & 100 & 100 & 100 & 100 & 100 & 100 & 100 & 100 & 200 \\
\hline
\end{tabular}

Tab. 1 : Analyse anthracologique du Rajal del Gorp en nombre de fragments par taxon et par période.

Tab. 1: Charcoal analysis of Rajal del Gorp: absolute frequencies of taxa for each archaeological period. 
lorsque trois espèces sont possibles : A. campestre, A. monspessulanum ou A. opalus. En effet, A. opalus se distingue de $A$. campestre par la présence de cellules marginales carrées dans les rayons, critère qui a été observé sur quelques échantillons.

La plupart des UF ont fait l'objet de 100 identifications de charbons de bois, un peu moins pour l'UF 107 et jusqu'à 200 pour l'UF 15 (tab. 1). Ce sont des quantités minimales. En effet, en anthracologie, l'effectif qui optimise le nombre de taxons rares obtenus, mais surtout garantit la stabilisation des fréquences des taxons, varie de 50 à 300 charbons de bois, mais par sécurité on retient 250 (Chabal, 1997). L'optimum est fonction de la fréquence du, ou des taxons les plus fréquents et non de la richesse spécifique, c'est pourquoi pour juger de celleci, il est inutile de tracer les courbes effort-rendement pour chaque UF (Chabal, 1997). Dans le cas présent, la composition forestière - comprenant deux taxons dominants, totalisant régulièrement plus de $80 \%$ des fréquences, et peu de taxons moyennement représentés explique en partie la pauvreté taxinomique. Il aurait fallu des effectifs bien plus élevés pour faire apparaître d'autres taxons rares. Nos résultats paraissent pourtant exploitables quant aux proportions entre espèces, du fait de la cohérence paléoécologique des spectres de fréquence successifs. En outre, la richesse spécifique relativement faible des échantillons, en partie liée au nombre un peu faible de fragments étudié par UF, a pu dépendre aussi du mode de fréquentation de la grotte et/ou d'une végétation forestière très ouverte et réellement pauvre (les résultats anthracologiques des autres sites du Causse ne révèlent en effet qu'une faible diversité de taxons, voir 5.4).

\section{4 - RÉSULTATS}

Le diagramme anthracologique du Rajal del Gorp (fig. 7) présente les variations des proportions des essences identifiées par phases chrono-culturelles. Les taxons sont ordonnés selon leurs affinités écologiques. Des intervalles de confiance (à $95 \%$ ) donnent la signification statistique des proportions des taxons. Le diagramme est divisé en quatre phases chrono-culturelles qui serviront de trame à notre interprétation. La première recouvre les périodes anciennes correspondant au Mésolithique. Un hiatus durant le Mésolithique moyen et/ ou final la sépare de la seconde, qui va du Néolithique ancien au premier âge du Fer ; la troisième correspond à la période d'occupation du sanctuaire durant La Tène finale et la quatrième comprend les Haut et Bas-Empire, jusqu'à l'abandon du site.

Les résultats mettent en évidence des dynamiques forestières qui peuvent être discutées au regard des autres données disponibles sur le Larzac et aux environs. On compte une vingtaine de taxons, pour un minimum de 16 essences. Les deux essences dominantes sont Quercus deciduous, le chêne à feuillage caduc et Pinus type sylvestris, le pin de type sylvestre.

Au début du diagramme, au Mésolithique, le pin de type sylvestre totalise environ $90 \%$ des fréquences. Pour des raisons climatiques, il s'agit très probablement du pin sylvestre, dénomination que nous employons dans la suite du texte, même si on ne peut pas le distinguer par son bois du pin noir de Salzmann, actuellement présent à l'état relictuel sur la bordure sud du causse (Roiron et al., 2013). En revanche, Pinus mugo subsp. mugo et P. mugo subsp. uncinata (également non distingués par leur bois), montagnards, sont écartés. Le pin sylvestre est une essence pionnière, adaptée à des températures fraîches, certainement déjà développée aux périodes précédentes du Mésolithique. On peut imaginer, à cette époque, une pineraie claire, car en peuplement quasiment pur, le pin sylvestre ne s'accommode que de peuplements peu denses, garantissant un bon éclairement.

Le chêne caducifolié est présent en très faibles taux dès le début du diagramme, au Mésolithique, accompagné de l'érable de Montpellier. Cette espèce compagne du chêne pubescent, héliophile ou de semi-ombre, xérophile, un peu plus abondante que le chêne, a peut-être précédé celui-ci dans les pinèdes claires. Quoi qu'il en soit, ce sont certainement les prémices de la mise en place de la chênaie caducifoliée, dont l'expansion se situe plus tardivement, au Mésolithique, absent du diagramme.

Du Néolithique à la Tène finale, la pinède va être colonisée par les feuillus dans une dynamique désormais classique, due au réchauffement du début de l'Holocène, dominée par le chêne à feuillage caduc, qui comprend au moins le chêne pubescent puisqu'on sait qu'il était présent dans la vallée du Tarn dès le Pléistocène supérieur, d'après les empreintes de feuilles (Quercus cf. pubescens) retrouvées dans les travertins du Chenil et du Réservoir (fig. 1) (Bazile E. et al., 1977 ; Vernet, 1985). Dès le dernier niveau du Néolithique ancien, le chêne atteint déjà $60 \%$ des fréquences du diagramme, constituant avec le pin une forêt mixte. Les espèces compagnes, appartenant à la chênaie sont principalement le buis, l'érable de Montpellier, l'érable champêtre et le noisetier. Malgré le fort développement de la chênaie sur le plateau, le pin sylvestre reste présent tout au long de la séquence, avec des taux qui ne descendront guère au-dessous de $10 \%$ en fin de séquence.

L'apparition d'essences héliophiles comme les Rosaceae Maloideae et Prunoideae - dont, très probablement, Prunus spinosa (prunelier) identifié dans les charbons de bois et Prunus spinosa/avium/cerasus identifié par la carpologie (Demierre et al., 2015) - le noisetier, mais aussi le buis (qui, à ces altitudes, est une essence de lumière), dénoterait une légère ouverture du paysage à partir du dernier niveau du Néolithique ancien. Le chêne sempervirent est absent du diagramme (seule une identification de Quercus au genre dans l'UF 429 pourrait appartenir à cette espèce), sa présence dans les causses méridionaux étant pourtant signalée vers 6000 B.C. (Krauss-Marguet, 1981 ; Vernet, 1985), à de très faibles taux et de façon sporadique.

Durant la période laténienne, le pin continue de régresser, atteignant des fréquences très faibles (moins de $10 \%$ ). Les essences caractéristiques de différents milieux, de sous-bois, de lisière, voire de bosquets ouverts ne paraissent pas plus abondantes et diversifiées. 


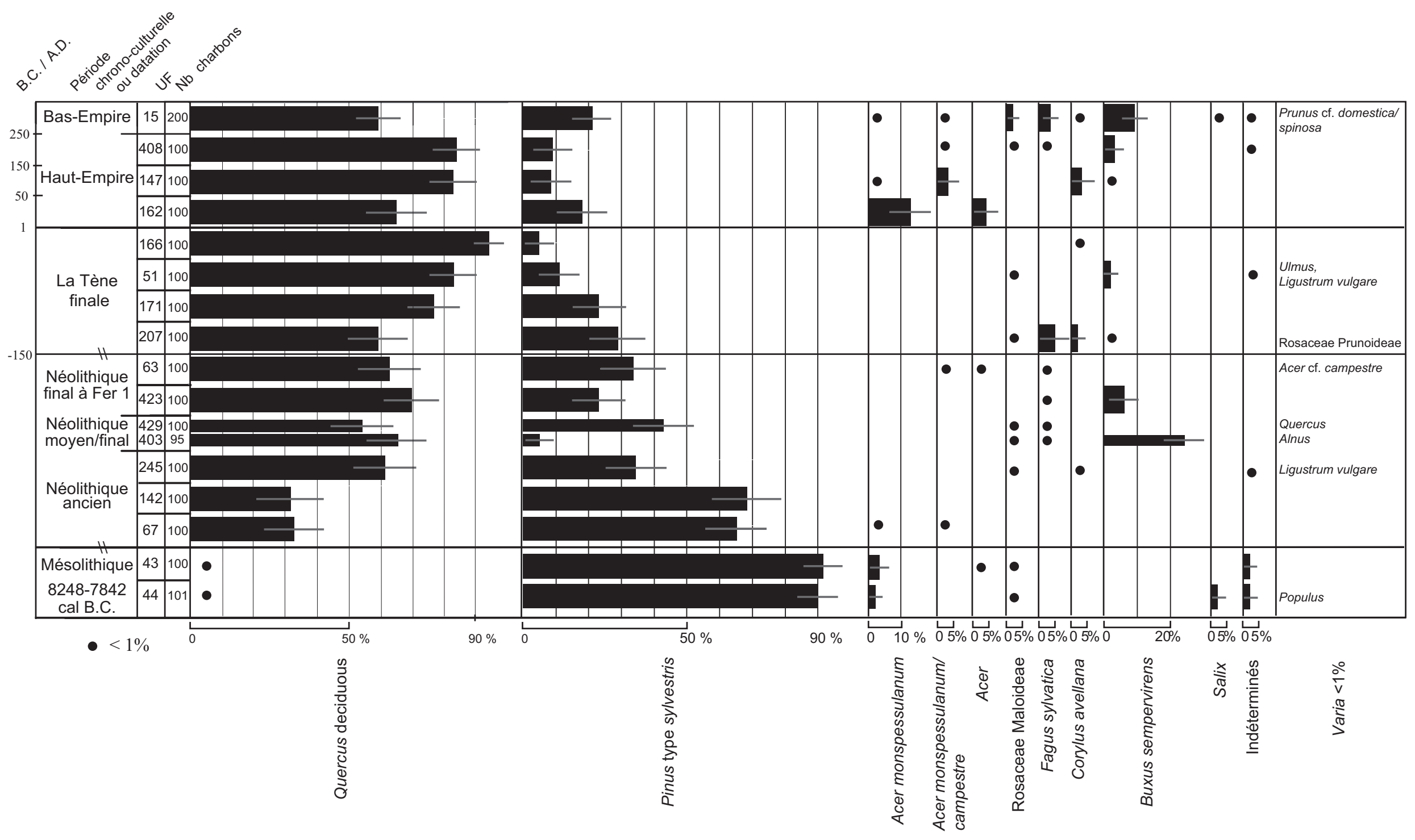

Fig. 7 : Diagramme anthracologique du Rajal del Gorp, avec intervalles de confiance à $95 \%$.

Fig. 7: Charcoal analysis diagram of Rajal del Gorp, $95 \%$ confidence intervals. 
Le hêtre, autour de $5 \%$ ou moins, dès le Néolithique moyen / final et jusqu'aux périodes laténienne et galloromaine, se maintient, mais ne paraît pas se développer sur le causse. La question de cette présence peu abondante et sporadique mérite d'être largement discutée (voir 5.5).

Durant le Haut et Bas-Empire, nous sommes ainsi en présence d'une forêt mixte mature, puisque dominée par le chêne pubescent, associée, en très faibles proportions (10\% pour le buis), à des essences de milieux ouverts et à de faibles fréquences de hêtre.

Nous discuterons des particularités paléoécologiques du Causse du Larzac, à travers ces résultats, afin d'essayer de définir dans quelle mesure cette dynamique forestière serait spontanée, d'origine purement climatique, ou due aux activités humaines. D'autre part, au regard de la dynamique paléoenvironnementale expliquée par les essences majoritaires, nous nous pencherons sur le cas particulier du hêtre, taxon montagnard.

\section{5 - DISCUSSION}

5.1 - LA RECONQUÊTE FORESTIÈRE POSTGLACIAIRE SUR LE CAUSSE DU LARZAC, QUELLES PARTICULARITÉS ET SIMILITUDES AVEC LE CONTEXTE MÉRIDIONAL ?

L'essor de la pineraie de pin sylvestre au Mésolithique au Rajal del Gorp est dû à la reconquête forestière sous l'effet du réchauffement climatique postglaciaire. Mais il existe de nombreuses variations micro-régionales de ce schéma en anthracologie. Pour beaucoup de sites, le stade pionnier de reconquête forestière est marqué par une formation pré-steppique de climat semi-aride froid (Quézel et al., 1980) avec un mélange de genévrier et de pin durant tout le Mésolithique. C'est le cas à la Poujade sur le Causse Noir voisin (KraussMarguet, 1981) ou, à plus basse altitude, à l'Abeurador dans l'Hérault durant le Mésolithique moyen (Heinz \& Thiébault, 1998), ce site révélant d'ailleurs trois espèces de genévrier (Vaquer \& Ruas, 2009). Les analyses polliniques de St-Sauveur (Hérault) sur le littoral (Puertas, 1998) montrent également une prédominance des pins et une occurrence régulière de genévrier avant l'expansion du chêne, dès le Mésolithique. Il en est de même sur différents sites provençaux de basse altitude (Triat-Laval, 1979). Dans le Sud-Ouest de la France, la séquence de la Cova de l'Esperit à $150 \mathrm{~m}$ d'altitude dans les Pyrénées orientales montre la dominance du genévrier et du nerprun / filaria au Mésolithique, puis du chêne vert, de l'olivier et du buis mais les pins n'y ont qu'un rôle mineur, au Chalcolithique, peut-être en raison de particularités thermiques (Solari \& Vernet, 1992). Les diagrammes polliniques issus de carottages, les plus proches du Rajal del Gorp, sont situés sur le Mont Lévézou (Beaulieu (de) 1981 ; Faure, 2012) ou le Mont Lozère (Beaulieu (de) \& Gilot, 1972 ; Reille, 1990 ; Pulido Avila, 2006), révélant des dynamiques postglaciaires de plus haute altitude et en milieu humide, différentes de celle du Larzac. L'amé- lioration climatique holocène s'accompagne souvent de la récession du pin (qui a peut-être joué un rôle antérieurement), au profit d'une végétation arboréenne où se succèdent des feuillus tels que Corylus, Quercus, Betula et Fagus. Pour Reille (1990), l'extension du pin sylvestre sur le Mont Lozère n'est liée qu'à l'ouverture agropastorale historique de la hêtraie.

A. Henry a réalisé l'étude anthracologique de plusieurs sites sur d'autres causses (Henry, 2011 ; Henry et al., 2013). En Aveyron, le pin n'est abondant qu'au Clos de Poujol, situé sur le Causse de Séverac à $860 \mathrm{~m}$ d'altitude, et dominera largement le chêne jusqu'au Néolithique ancien, probablement à cause de conditions climatiques fraîches, avec moins d'influences méridionales et une altitude plus élevée qu'aux Usclades (Théry et al., 1996), sans pouvoir totalement écarter l'intrusion de charbons de bois datant du Mésolithique. Sur le Causse de Gramat dans le Lot, entre 250 et $330 \mathrm{~m}$ d'altitude et sous influence atlantique marquée, les sites des Fieux, des Escabasses et du Cuzoul de Gramat sont marqués par un essor très précoce de la chênaie, majoritaire dès le Mésolithique ancien. Dans un contexte climatique régional océanique, plus frais et humide que sur le Causse du Larzac, l'effet de l'exposition et de conditions localement plus xériques, mais aussi la probable proximité de zones refuges du chêne caducifolié, seraient ici les facteurs ayant favorisé le chêne, par rapport aux régions méditerranéennes. Pour ces trois derniers sites, la chênaie progresse au détriment d'une forte proportion de Prunoideae et Maloideae, en récession, interprétées comme des stades préforestiers du domaine bioclimatique de la chênaie. Ils sont observables entre les niveaux 6 et $5 \mathrm{~b}$ des Escabasses respectivement datés de $7521-7145$ cal. B.C. (Ly-12238) et 6204 - 5886 cal. B.C. (Ly-10938) (Henry et al., 2013).

La séquence de Troubat, à $540 \mathrm{~m}$ d'altitude dans les Hautes-Pyrénées, montre que des phases à Prunoideae et Rhamnaceae, plus précoces encore, s'insèrent entre la récession du genévrier et la succession pin sylvestre / chênaie, pouvant signifier des espaces steppiques encore très ouverts et, pour ce site, froids (Heinz \& Barbaza, 1998).

Mais, dès le Néolithique, l'appartenance des Rosaceae à une succession progressive ou régressive est discutable, puisque ces essences sont également liées à l'éclairement et pourraient révéler une ouverture anthropique précoce du paysage.

De tous ces sites, il ressort que, selon les conditions micro-régionales ou l'altitude, il faut envisager que le bouleau ou les Rosaceae aient pu jouer un rôle similaire à celui des genévriers, dans la transition entre les stades forestiers postglaciaires et l'installation de la chênaie, mais que des fruticées héliophiles d'origine anthropique ont également pu se développer précocement aux détriments de la chênaie.

Toujours est-il qu'aucun élément préforestier n'apparaît au Rajal del Gorp, pour les niveaux dont nous disposons. Des Rosaceae Maloideae et Prunoideae sont présentes en filigrane tout au long de la séquence, sans qu'aucune augmentation ne soit visible dans les niveaux 
du Mésolithique moyen / final et du Néolithique présents dans le diagramme. Dans l'environnement du Rajal del Gorp, le développement du chêne s'est probablement fait en l'absence de formation préforestière ou présteppique. D'ailleurs, aux Usclades, on observe qu'aux niveaux sauveterriens [niveau 4 daté de 7455-7066 cal. B.C. (Gif. 8744)], où le pin sylvestre domine à pratiquement $90 \%$, succède directement le Néolithique ancien caractérisé par le chêne décidu $(70 \%)$, le pin $(10 \%)$ et de très rares Prunoideae et autres essences héliophiles (Théry et al., 1996). À la Poujade, il en est de même (si l'on excepte un niveau riche en Prunus type dulcis dont l'anatomie peut le faire confondre avec Prunus spinosa). Il se pourrait donc qu'il n'ait pas existé de stade préforestier sur le Larzac et le Causse Noir. Il est possible que cette spécificité, par rapport au Causse de Gramat par exemple, tienne à l'absence, à proximité, de semenciers d'espèces préforestières. Cependant, le caractère très implanté du pin sylvestre sur le Larzac, avec de forts taux dès le début de la séquence mésolithique du Rajal del Gorp et des Usclades, constitue une différence avec les sites précédemment évoqués, rappelant l'appartenance du plateau à la série mixte du pin sylvestre et du chêne pubescent/sessile. La perduration des pins sur le Larzac jusqu'à la période gallo-romaine et certainement au-delà, diffère aussi d'avec les séquences mésoméditerranéennes connues, dans lesquelles les feuillus se substituent complètement aux résineux pour des raisons thermiques. Le pin sylvestre, en particulier, est peu adapté aux basses altitudes même si des occurrences faibles au Subatlantique sont possibles en situations protégées (Roiron et al., 2013). Il est probable que la perduration des pins sur le Larzac soit due aux effets thermiques conjugués de l'altitude et des influences atlantiques. En effet, le sommet des plateaux caussenards appartient à la base de l'étage montagnard. De plus, l'inclinaison des plateaux vers l'ouest permet la pénétration des vents océaniques, le Causse du Larzac percevant cependant les masses d'air tièdes et humides de la Méditerranée (IFN, 1995). Cette situation d'interface climatique, conjuguée à une faible perturbation du milieu et à une présence extensive du pin sylvestre dans le passé, pourrait expliquer sa perduration dans le temps en dépit du redoux climatique holocène. La chênaie se serait développée sous la protection d'une pineraie claire, sans stade préforestier protecteur des plantules.

\section{2 - L'EXPANSION DE LA CHÊNAIE CADUCI- FOLIÉE SUR LE CAUSSE DU LARZAC}

Les synthèses sur la flore préhistorique des Grands Causses (Vernet, 1968, 1972, 1985) situent l'installation de la chênaie autour de 7000 B.C., voire un peu plus tôt à La Poujade (niveau $10 \mathrm{C}$ daté autour de 8417-7601 cal. B.C.), et sa prédominance autour de 3500-3000 B.C., soit pas avant le Néolithique final (Vernet, 1985) (fig. 6).

D'après le diagramme du Rajal del Gorp et les niveaux datés dont nous disposons, il semble que les pinèdes soient largement dominantes sur le Larzac durant le Mésolithique et que le développement de la chênaie soit très important dès le début du Néolithique, puisque le chêne passe d'environ $30 \%$ à $60 \%$ au cours du Néolithique ancien, pour n'augmenter à nouveau qu'à $\mathrm{La}$ Tène finale (de 60 à plus de $90 \%$ ). On ne peut exclure un début de développement de la chênaie dès le Mésolithique final, absent du diagramme. Quoi qu'il en soit, le processus de substitution semble légèrement plus tardif au Rajal del Gorp que sur les autres sites évoqués qui comprennent une dynamique du pin. Ainsi, le développement des chênaies est déjà important au Mésolithique moyen à La Poujade ou à l'Abeurador. De même, les diagrammes polliniques du Sud de la France enregistrent entre 25 et $50 \%$ de pollens de chêne à feuillage caduc, déjà vers 9000 B.P. (correspondant à la fin du Mésolithique ancien, vers $8200 \mathrm{cal}$. B.C.) (Brewer et al., 2002). Sous influence atlantique, la chênaie est même présente sur le Causse de Gramat dès la fin de l'Azilien (fin de l'Épipaléolithique), peut-être grâce à la proximité de refuges glaciaires (Henry et al., 2013).

L'explication d'un développement un peu plus tardif des chênaies sur le causse du Larzac est-elle liée à l'absence de semenciers ? En effet, l'arrivée du chêne caducifolié correspond à une expansion spontanée de son aire de répartition depuis des zones refuges, au moment du réchauffement climatique. Comme l'ont montré les études croisées du pollen et de l'ADN (Brewer et al., 2002 ; Petit et al., 2002), la forêt tempérée se serait diffusée depuis des refuges glaciaires principaux tels que les péninsules ibérique, italique et les Balkans, et secondaires, qui ont pu jouer un rôle dans sa rapide diffusion dans certaines localités, telles que les régions situées sous influence méridionale proches des principaux foyers de diffusion. À proximité du Larzac, le chêne était pourtant présent dans la vallée du Tarn au Pléistocène supérieur (voir 4.) qui a pu constituer une zone refuge. La raison de l'arrivée tardive du chêne caducifolié sur le Causse du Larzac, au plus tôt au Mésolithique ancien / moyen, est probablement en partie thermique, due à l'altitude du Causse du Larzac. Le développement du chêne est rendu possible par le réchauffement climatique, puis obéit à une dynamique d'expansion spontanée au début du Néolithique au détriment des pinèdes, désormais moins adaptées au climat. Les coupes ont également pu participer à favoriser l'extension du chêne, adapté à y résister, contrairement au pin qui régénère par semis. En revanche, entre la fin du Néolithique ancien et la Tène finale, le taux de chêne est assez stable suggérant un équilibre entre sa dynamique spontanée et l'action humaine. La chênaie se maintiendrait alors sous forme de taillis.

\section{3 - LA PLACE DES ESPÈCES MÉSO-HYGRO- PHILES DANS LE PAYSAGE}

Le frêne, l'orme, l'érable champêtre, le saule, l'aulne et le peuplier, communément attribués à des milieux alluviaux et/ou de ripisylve sont présents en très faible fréquences et sporadiquement dans les niveaux étudiés. Or, aucune source, ni cours d'eau permanent ne sont présents à proximité du site et aucun indice ne nous 
permet d'en soupçonner dans le passé. Bien que préférant les sols à bilan hydrique favorable, l'érable champêtre, le frêne et l'orme peuvent éventuellement se développer dans la chênaie. La présence de saule (en début et en fin de diagramme), de peuplier et d'aulne, indique par contre un milieu humide. Leur présence pose donc le problème de l'approvisionnement en eau sur cette partie du Larzac. D'après la carte IGN et la carte géomorphologique de Ambert (1982), il existe un cours d'eau temporaire près du site, à environ $500 \mathrm{~m}$. Encore actuellement sur le Larzac, on peut observer très localement des espèces exigeantes en humidité éloignées de tout cours d'eau, mais qui profitent de circulations d'eau superficielles (bas de pente, vallons, fossés), sans oublier, sur cette partie du Larzac, des dolines naturelles remplies d'argile qui forment des mares temporaires. Enfin, on ne peut pas exclure que ce bois ait été collecté beaucoup plus loin, sur les bords du Tarn ou de la Dourbie.

\section{4 - UNE PRESSION ANTHROPIQUE MODÉRÉE TOUT AU LONG DE LA FRÉQUENTATION DU SITE}

L'absence de stades préforestiers composés d'essences héliophiles, à la transition Mésolithique / Néolithique a été notée au Rajal del Gorp, aux Usclades et à la Poujade, interprétée comme l'implantation directe des chênes au sein des pinèdes (voir 5.1). On remarque qu'il n'existe pas, non plus, de diversification importante des essences au cours de la séquence. Si une diversité de biotopes a existé sur le Larzac, elle pouvait être située hors de l'aire de collecte du bois du Rajal del Gorp, aire réduite en raison de durées ou densités d'occupation faibles. La chênaie ne paraît que très peu affectée par la pression anthropique. Le noisetier, espèce d'ouverture rattachée à la chênaie, est très discret. Les marqueurs d'ouverture du paysage et de pâturage restent en assez faible proportion : les Prunoideae sont sporadiques, et seul le buis atteint 5 à $10 \%$, et même $25 \%$ à partir du Néolithique moyen / final. La fréquence élevée du buis dans l'UF 403 signe peut-être les débuts de l'expansion de cette espèce, non consommée par les troupeaux. Toutefois, il n'atteint plus de tels taux par la suite. Il n'est pas exclu qu'une collecte sélective de buis comme litière, pratique bien connue dans les temps historiques (Durand-Tullou, 2009 ; Olivier, 2012), soit en partie la cause d'un tel pourcentage. Cette fréquence isolée serait alors un artefact dû à son utilisation comme litière, et son réemploi comme bois de feu. Sur d'autres sites contemporains du Larzac, le buis atteint $60 \%$ à l'âge du Bronze. Ainsi, à la Poujade en Aveyron, et à Font-Juvénal dans l'Aude (Thiébault \& Vernet, 1992), c'est dès le Néolithique moyen que le buis et les espaces ouverts vont se substituer pour partie à la chênaie.

D'autres essences, comme les chênes sempervirents ou le filaire, très développées sur les sites méridionaux de l'étage mésoméditerranéen à l'âge du Fer et à la période romaine, ne sont pas présentes ici. Le chêne sempervirent (type chêne vert) est absent de nos identifications (si l'on excepte un fragment de chêne déterminé au rang du genre), alors que quelques fragments en sont présents à partir du Néolithique à la Poujade, située en versant sud. Actuellement, le chêne vert n'existe plus qu'à basse altitude, sur le pourtour du plateau ainsi que sous forme de rares colonies, le long du Tarn et de la Jonte (fig. 1) (Vernet, 1985). Ces résultats confirment la faible adaptation du chêne vert aux conditions thermiques du plateau durant les périodes étudiées.

\section{5 - A-T-IL EXISTÉ UNE HÊTRAIE SUR LE LARZAC ? SYNTHÈSE ÉCOLOGIQUE ET PALÉO- ENVIRONNEMENTALE}

\subsection{1 - Le hêtre dans les séquences paléobotaniques du Causse du Larzac et de ses marges}

Concernant l'ancienneté de la présence du hêtre et son étendue sur le Causse du Larzac dans le passé, l'étude du Rajal del Gorp vient combler une lacune chronologique, puisqu'aucun site comprenant des périodes situées entre l'âge du Fer et l'Antiquité n'avait été étudié en anthracologie auparavant.

Les hypothèses anciennes selon lesquelles le Larzac était originellement peuplé de hêtraies, du fait de son extension observée à la fin du $\mathrm{xx}^{\mathrm{e}} \mathrm{s}$. (Prioton, 1970), ont été réfutées par la paléobotanique. Vernet (1975), notamment, avait tout à fait perçu sa faible adaptation au Causse du Larzac, contrairement au chêne pubescent et au pin sylvestre. Et, constatant que les sites préhistoriques du Causse du Larzac ne révélaient aucune couverture de la hêtraie entre le Paléolithique et le Néolithique, Vernet en déduisait qu'aucune hêtraie n'avait pu exister sur le Larzac avant le Subboréal (Vernet, 1975). Cependant, l'absence de donnée anthracologique pour les périodes récentes laissait jusqu'alors la possibilité de supposer un développement tardif de cette essence, après l'âge du Bronze (à partir de 1000 B.C.) sur le Larzac (Vernet, 1985) et même son défrichement précoce (Vernet, 1973). En effet, son apparition à cette période avait été observée par Vernet (1985) sur le Causse Méjean, à des altitudes supérieures à celles du Larzac, autour de 1100-1200 m. Par ailleurs, dans les secteurs de la Margeride, du Lévézou, du Mont Lozère, des Cévennes ou dans l'Aubrac, le hêtre prend de l'ampleur dans les diagrammes palynologiques, évoluant parfois en hêtraie-sapinière à partir de la fin du Néolithique pour atteindre le maximum de son expansion au Subboréal avant de disparaître sous l'effet des coupes et de l'anthropisation (Lemée 1953 ; Beaulieu (de) \& Gilot 1972 ; Beaulieu (de) 1974 ; Reille, 1990 ; Pulido Avila, 2006 ; Faure, 2012 ). On comprend qu'il était tentant d'envisager un scénario proche sur le Causse du Larzac, en dépit de sa latitude méridionale et de son altitude modérée (700 à $900 \mathrm{~m}$ ). Mais avant d'examiner l'apport du Rajal del Gorp à l'histoire du hêtre, il importe de bien prendre en compte son écologie.

\subsection{2 - Écologie du hêtre dans le sud du Massif central et en marge de son aire de répartition}

En raison des apports de la paléobotanique (voir 5.5.1), la question de la présence et du développement de hêtraies sur le Larzac dans le passé a été soulevée 
à maintes reprises. La présence actuelle du hêtre est observée surtout sur la bordure sud du plateau, la plus arrosée avec $1000 \mathrm{~mm}$ de précipitations annuelles, mais aussi au centre du plateau à une altitude avoisinant les 800-850 m (Vernet, 1972). Ces boisements sont-ils le reliquat de hêtraies originelles plus étendues, telles que celles des reliefs alentour révélées par la palynologie, ou le hêtre y a-t-il toujours appartenu à des milieux dominés par la chênaie supraméditerranéenne ? Dans un cas comme dans l'autre, était-il bien adapté, au point de prendre quelque importance?

Plusieurs botanistes et phytosociologues se sont intéressés à la présence du hêtre dans les zones méridionales. Braun-Blanquet (1970) souligne que les hêtraies des causses méridionaux ne présentent pas d'unité au plan phytosociologique. Il rattache le hêtre à deux types de formations :

- les unes, proches des hêtraies européennes, rattachées au Fagetum gallicum buxetosum, qui apparaissent dans les stations les plus fraîches du Larzac, les ubacs très prononcés au pied des falaises, les creux rocheux, les zones humides, et forment actuellement des hêtraies isolées au milieu de la chênaie de chêne pubescent, avec des hêtres de plusieurs centaines d'années et des chênes en sous-bois ;

- les autres, appartenant au Querco-Buxetum fagetosum, secondairement dominées par le hêtre, mais rattachées à la chênaie, croissant entre 750 et $850 \mathrm{~m}$ et souffrant de l'emprise humaine (présentes notamment au nord du Caylar).

Bien que ces lambeaux de hêtraies soient bien développés, Braun-Blanquet (1970) note que leur extension est limitée par la sécheresse estivale et que c'est avant tout la chênaie qui tend à gagner de l'espace. On peut en effet douter de l'aptitude du hêtre à s'étendre beaucoup. Il s'agit d'une essence qui rejette très mal de souche hors de l'étage montagnard (Rameau et al., 2008) et son dynamisme est moindre en limite de son aire de répartition. En marge du domaine méditerranéen, les hêtraies se développent dans des conditions écologiques particulières, différentes de celles des zones plus septentrionales (Thiébaut, 1982). Les conditions stationnelles sont alors déterminantes dans la présence ou non du hêtre au sein de ces zones du fait que le climat ne lui est pas optimal. Les facteurs qui entrent en jeu sont d'ordre édaphique, topographique et anthropique (E Silva, 2010).

Les facteurs de compensation de la contrainte climatique régionale dans le domaine méditerranéen se présentent sous plusieurs formes, qui peuvent se cumuler pour pallier la sécheresse. On compte, parmi elles l'humidité édaphique, qui peut être accrue par la présence d'une forte épaisseur d'humus ou la présence de réserves d'eau dans le sous-sol ou en situation riveraine. À ce titre, Vanden Berghen (1963) signale la présence d'une hêtraie riveraine de belle venue sur les basses terrasses du Tarn et de la Dourbie, à $350 \mathrm{~m}$ d'altitude, dans laquelle le hêtre domine, accompagné d'espèces mésophiles (tilleul à grandes feuilles, érable à feuilles d'obier). Le rôle des apports organiques nutritifs pour la hêtraie par les crues de la rivière semblent également participer à son maintien. Plus xériques, néanmoins, que la hêtraie calcicole à buis du fait de sols arénacés, ces boisements ont évolués puisqu'en 1987, Durand (1998) y note une différence de composition ; le hêtre étant largement mélangé aux essences de la chênaie supra-méditerranéenne de chêne pubescent, avec présence du pin sylvestre et du châtaignier. Durand (1998) souligne l'importance du facteur microclimatique d'adret ou d'ubac et du phénomène d'inversion d'étage dans la caractérisation des relevés floristiques.

Sous influence méditerranéenne, le hêtre se comporte comme une ombrophile se développant dans les sous-bois de la chênaie, profitant à la fois de l'effet protecteur du boisement contre l'évapotranspiration et de l'épaisseur des sols. Les brouillards peuvent également compenser l'aridité estivale et ont une influence sur la répartition des boisements de hêtre, qui généralement n'existe dans le sud du Massif central qu'au-dessus de $800 \mathrm{~m}$ (Flahault, 1893 ; Thiébaut, 1982). Enfin, le régime sylvicole apparaît comme un facteur limitant important lors des traitements des boisements en taillis. En effet, si le hêtre supplante le chêne dans les vieilles chênaies, il possède une faible capacité à rejeter de souche et disparaît progressivement des taillis, le chêne pubescent remplaçant le hêtre dans les boqueteaux surexploités (Vanden Berghen, 1963 ; Peltier et al., 1997 ; Emborg et al., 1998 ; Tognetti et al., 1998 ; E Silva, 2010).

Ainsi, le Causse du Larzac semble constituer une " zone d'épreuve du hêtre ", en limite avec celle du chêne (Flahault, 1901). On y observe des mélanges de végétations, souvent hétérogènes, aux limites floues car très sensibles aux microclimats. Notons, par exemple, la présence du hêtre à $500 \mathrm{~m}$ d'altitude sur le versant sud du Larzac, au contact du chêne vert (Durand \& Vernet, 1987) qui illustre, encore une fois, la relative plasticité de cette essence en marge de son aire de répartition.

Au regard des enregistrements paléobotaniques et de l'écologie du hêtre, comment comprendre son histoire et sa place sur le Larzac? De quand son relatif développement peut-il dater?

\subsection{3 - Paléoécologie du hêtre sur le Causse du Larzac : un développement limité}

L'étude du Rajal del Gorp nous éclaire pour trancher ce débat. Les faibles taux de hêtre à partir du Néolithique moyen / final attestent de sa présence bien avant le Subboréal, contrairement à ce qui avait été avancé. À l'échelle régionale, le hêtre aurait pu diffuser depuis des refuges pléniglaciaires situés sur les marges de la vallée du Rhône, sa courbe pollinique ne devenant continue dans le sud de la France qu'au Préboréal (Delhon \& Thiébault, 2005).

Son implantation sur le Larzac est certainement due au développement en cours de la chênaie calcicole et à la maturation en cours de ce boisement, qui offrait son effet protecteur. Mais, pour autant, l'étude du Rajal del Gorp montre que le hêtre n'a pas connu un développement 
important au nord du plateau du Larzac du Mésolithique ancien/moyen à la période gallo-romaine. Plus encore, on peut en déduire que si les chênaies avaient existé à l'état de futaie mature sur toute la séquence du Rajal del Gorp, il est probable que le hêtre s'y serait davantage développé. Rappelons que Vanden Berghen (1963) a explicité l'affinité entre la hêtraie et la chênaie de chêne pubescent sur les Grands Causses : « le hêtre supplante le chêne dans les vieilles chênaies, et le chêne pubescent remplace le hêtre dans les boqueteaux surexploités ». Ce schéma correspond à nos observations. Nous pouvons penser que la forêt de chêne pubescent en plein développement a été transformée de futaie en taillis au cours du Néolithique par les activités humaines dont l'exploitation devait être défavorable à un développement plus important du hêtre.

Ainsi, le hêtre se serait implanté à la faveur de la protection des chênaies sur substrat calcaire en dépit de la sécheresse estivale sur le Causse du Larzac, dès le Néolithique moyen / final. Le hêtre en limite climatique aurait été, au départ, favorisé par le développement de la chênaie puis, au fur et à mesure de l'ouverture de celleci, maintenu en peuplements localisés à la faveur des stations les plus fraîches ou les plus arrosées, tels que les vallons protégés de la sécheresse estivale, la bordure méridionale, ou les zones altitudinales comme c'est le cas encore actuellement.

Les feuilles d'if retrouvées dans les niveaux laténiens (Demierre et al., 2015) attestent de la présence de cette essence sur les Causses à cette période. L'if est actuellement absent du plateau. Son écologie le situe de préférence dans les hêtraies (même thermophiles) mais il peut être présent dans les chênaies de chêne pubescent (Rameau et al., 2008). Probablement peu répandu dans le passé, l'if a pu se retrouver associé au chêne et au hêtre dans les stations à humidité atmosphérique élevée.

\section{6- CONCLUSION}

L'étude anthracologique du Rajal del Gorp montre une succession forestière postglaciaire spontanée du Mésolithique ancien/ moyen à la période gallo-romaine. Le stade pionnier de reconquête forestière au Mésolithique (Préboréal / Boréal) est caractérisé par des pineraies, très probablement de pin sylvestre en peuplements presque purs, sans stade intermédiaire riche en genévrier ou bouleau en particulier. En début de séquence, de très faibles taux de chêne caducifolié et la présence de l'érable de Montpellier suggèrent le début de la diffusion du chêne. Cette extension depuis les refuges méridionaux est tardive au regard des données régionales qui révèlent un développement de la chênaie déjà avancé au Mésolithique ancien ou moyen dans le sud de la France. Aucun stade préforestier lors de l'implantation de la chênaie, notamment de Rosaceae, n'est identifié, peut-être du fait de l'absence locale de semenciers de ces espèces héliophiles. Le développement de la chênaie supra-méditerranéenne dominée par le chêne caducifolié (très probablement le chêne pubescent) au sein de pinèdes claires conduit à une forêt mixte dans laquelle le chêne supplante le pin durant le Néolithique. Le chêne devient dominant dès le Néolithique moyen / final. Le pin se maintient à des taux d'au moins 5 à $10 \%$ durant toute la séquence. Il ne disparaît pas, contrairement à ce que l'on observe à l'étage mésoméditerranéen en Languedoc. Bénéficiant de sa situation de «premier occupant» implanté au cours de conditions climatiques rigoureuses qui le favorisaient, il a gardé une très bonne adaptation à la rigueur hivernale du Causse du Larzac.

La présence du hêtre en faibles proportions tout au long de la séquence, dès le Néolithique ancien et à des périodes jusque-là non renseignées à ce sujet (périodes laténienne et gallo-romaine), est désormais démontrée pour cette partie du Larzac. Le hêtre a certainement connu une importance un peu supérieure à celle actuelle sous l'effet protecteur de la chênaie, rapidement freinée par l'exploitation des taillis. Pour autant, l'hypothèse d'une extension de cette essence durant la Protohistoire, qui avait été proposée par d'autres auteurs, est réfutée, tout au moins pour la partie nord du causse. Les conditions climatiques, liées à une altitude insuffisante pour générer des nébulosités et des précipitations importantes, et les conditions édaphiques propres au Causse du Larzac ne lui ont permis de perdurer que de façon localisée et mineure.

Enfin, l'ouverture du milieu, telle qu'elle est perçue dans le sud de la France dès le Néolithique et avec une accélération à la Protohistoire, marquée par une apparition massive d'essences sempervirentes et héliophiles, n'est ici perceptible que par des taux de buis d'importance modérée, ce qui suppose une faible ouverture de la couverture forestière. Cette observation, alliée au faible développement du hêtre qui démontre au contraire l'absence de perduration de la futaie fermée de chêne, suggère la transformation de la chênaie en taillis denses par l'activité humaine, au moins au nord du Causse du Larzac et jusqu'à la période romaine. Le fort développement des landes à buis et genévrier au détriment de la chênaie, pour conduire finalement aux paysages déboisés que nous connaissons aujourd'hui, est donc postérieur à la période gallo-romaine.

L'existence de ces taillis de chêne caducifolié très matures vient tempérer ce que l'on retrouve sur les sites alentour, pour démontrer la juxtaposition de dynamiques différentes selon le climat local et l'histoire anthropique de chaque secteur des causses et, au final, l'existence de paysages en mosaïque à chaque époque.

\section{REMERCIEMENTS}

Ce travail a bénéficié du soutien financier de l'UMR-CNRS 5140 «Archéologie des Sociétés Méditerranéennes » et du LabEx ARCHIMEDE (PIA ANR11-LABX-0032-01). Nous remercions I. Figueiral-Rowe (INRAP, ISEM-UMR 5554) pour la traduction des textes en anglais, ainsi que L. Paradis (ISEM-UMR 5554) pour sa contribution à l'extraction des données cartogra- 
phiques et du D.A.O des cartes. Tous nos remerciements à P. Poirier (INRAP, ISEM-UMR 5554) et J.-E. Brochier (LAMPEA-UMR 7269) pour leur relecture.

\section{RÉFÉRENCES BIBLIOGRAPHIQUES}

AMBERT P., 1982 - Carte géomorphologique de la région de Millau $(1 / 25000)$.

BARBERO M., BONIN G. \& QUEZEL P., 1971 - Signification bioclimatique des pelouses écorchées sur les montagnes du pourtour méditerranéen, leurs relations avec les forêts d'altitude, Bulletin de la Société Botanique de France, 118, sup2, 17-56.

BARBERO M., MERLE (du) P., GUENDE G. \& QUEZEL P., 1978 - La végétation du Mont Ventoux. Revue d'écologie appliquée, supplément 1, 21-37.

BAZILE E., BAZILE F., \& VERNET J.-L., 1977 - Une flore associée à Picea abies (L.) Karsten dans les tufs calcaires pléistocènes des Grands Causses. Comptes Rendus de l'Académie des Sciences, Paris, 284 (D), 2211-2214.

BEAULIEU J.-L. (de), 1974 - Évolution de la végétation sur la bordure montagneuse cévenole au postglaciaire, d'après les pollens. Bulletin de la Société Languedocienne de Géographie, 8, 347-358.

BEAULIEU J.-L. (de), 1981 - Analyse pollinique de tourbières sur la marge occidentale et septentrionale des Causses. Paléobiologie continentale, 12, 117-130.

BEAULIEU J.L. (de) \& GILOT E., 1972 - Végétations holocènes du Mont Lozère : analyses polliniques et datages. Comptes Rendus de l'Académie des Sciences de Paris, 274, 1641-1644.

BEAULIEU (de) J.L., PONS A. \& REILLE M., 1982 - Recherches pollenanalytiques sur l'histoire de la végétation de la bordure nord du massif du Cantal (Massif Central, France). Pollen et spores, 24, 251-300.

BEAULIEU (de) J.L., PONS A. \& REILLE M., 1984 - Recherches pollenanalytiques sur l'histoire des Monts de Velay (Massif Central, France). Dissertaciones Botanicae, 72, 45-70.

BEAULIEU (de) J.L., PONS A. \& REILLE M., 1985 - Recherches pollenanalytiques sur 1'histoire Tardiglaciaire et Holocène de la végétation des Monts d'Aubrac (Massif Central, France). Review of Palaeobotany and Palynology, 44, 37-80.

BOTTOLLIER-CURTET M. \& MULLER S., 2008 - Dynamique et contexte passés du développement d'une tourbière méditerranéenne (Massif de l'Aigoual, France). Biologies, 332, 69-82.

BOULANT N. \& LEPART J., 2008 - La progression du Pin sylvestre et du Pin noir dans le paysage des Grands Causses : impact des activités humaines et des facteurs naturels. Revue Forestière Française, 60 (5), 603-614

BRAUN-BLANQUET J., 1970 - La végétation sylvicole des Causses méridionaux. Pirineos, 95, Jaca, 47-74.

BREWER S., CHEDDADI R., DE BEAULIEU (de) J.L., REILLE M. \& DATA CONTRIBUTORS, 2002 - The spread of deciduous Quercus Throughout Europe since the last glacial period. Forest Ecology and Management, 156, 1-3, 27-48.

BRONK RAMSEY C., 2009 - Dealing with outliers and offsets in radiocarbon dating. Radiocarbon, 51 (3), 1023-1045.

CHABAL L. 1997 - Forêt et sociétés en Languedoc (Néolithique final, Antiquité tardive), l'anthracologie, méthode et paléoécologie. Documents d'Archéologie Française, 63. Maison des Sciences de l'Homme, Paris, 189 p.

CHABAL L., FABRE L., TERRAL JF \& THÉRY-PARISOT I., 1999 - L'anthracologie. In: Ferdiere A. (Ed.), La botanique. Errance, Collection Archéologiques, Paris, 43-104.

CHAMPAGNE F., CHAMPAGNE C., NOVEL P. \& JAUZON P., 1990 - Le site préhistorique des Fieux a Miers (Lot). État actuel des recherches. Gallia Préhistoire, 32, 1-28.

COSTANTINI G., 1984 - Le Néolithique et le Chalcolithique des Grands Causses, Gallia-Préhistoire, 27, 121-210.

DELHON C. \& THIÉBAULT, 2005 - The migration of beech (Fagus sylvatica L.) up the Rhone: the Mediterranean history of a "mountain" species. Vegetation History and Archaeobotany, 14, 119-132.

DELIBRIAS G., GUILLIER M., \& LABEYRIE J., 1970 - GIF Natural Radiocarbon Measurements V. Radiocarbon, 12 (2), 421-443.

DEMIERRE M., avec la collab. de DURAND F., FOUCRAS S., GRUEL K., JENNY J. \& LIOTTIER L., 2015 -La grotte-sanctuaire rutène du Rajal Del Gorp. Bilan des fouilles récentes et particularités. In OLMER F., ROURE R. (éd.), Les Gaulois au fil de l'eau. Actes du 37e colloque international de l'AFEAF (Montpellier, 8-11 mai 2013), Ausonius, Bordeaux, 697-718.
DEMIERRE, M. (dir.) \& MENDIBURU F., 2011 - La grotte sanctuaire du Rajal del Gorp (Millau, Aveyron). Rapport de la campagne de fouille 2011 et synthèse des résultats des fouilles anciennes et récentes (2008-2011), fouille programmée triennale de juin 2011. SRA Midi-Pyrénées, $132 \mathrm{p}$.

DURAND A., 1998 - Les paysages médiévaux du Languedoc $\left(X^{e}-X I I^{e}\right.$ siècles), presses de l'université de Toulouse-le-Mirail, collection Tempus, mars 1998, $491 \mathrm{p}$.

DURAND-TULLOU A., 2009 - Un milieu de civilisation traditionnelle : le Causse de Blandas, Éditions du Beffroi, Montpellier, 520 p.

EMBORG, J., 1998 - Understorey light conditions and regeneration with respect to the structural dynamics of a near-natural temperate deciduous forest in Denmark. Forest Ecology and Management, 106, 83-95.

E SILVA D., 2010 - Écologie du hêtre (Fagus sylvatica L.) en marge sud-ouest de son aire de distribution. Thèse de doctorat de l'université Henri Poincaré, Nancy I, 192 P.

FAURE E., 2012 - «Hautes terres »: l'anthropisation des monts d'Aubrac et du Lévezou (Massif central, France) durant l'Holocène : approche palynologique des dynamiques socio-environnementales en moyenne montagne. Thèse de doctorat de l'Université Toulouse le Mirail, Toulouse II,

FLAHAULT C.H., 1893 - La distribution géographique des végétaux dans un coin du Languedoc. Imprimerie Ricard Frères, Montpellier, $180 \mathrm{p}$.

FLAHAULT C.H., 1901 - Les limites supérieures de la végétation forestière et les prairies pseudo-alpines. Revue Eaux et Forêts, 40, 385-401; 417-439.

FLAHAULT C.H., 1932 - Les Causses du Midi De la France. Bulletin de la Société Languedocienne de Géographie, 3 (3), 97-118.

FLAHAULT C.H., 1933 - «Les Causses du Midi De la France », Bulletin de la Société Languedocienne de Géographie, 4 (4), 153-184.

FLAHAULT C.H., 1934 - «Les Causses du Midi De la France », Bulletin de la Société Languedocienne de Géographie, 5 (1), 1-22.

GRUAT P., DEMIERRE M., avec la collab. de PUJOL J. ( $\dagger$ \& VERNHET A., 2015 - Un exemple original de dépôt en milieu humide : les grottes sanctuaires du territoire des Rutènes et de ses marges à la fin de l'âge du Fer. In OLMER F., ROURE R (éd.), Les Gaulois au fil de l'eau. Actes du $37^{e}$ colloque international de l'AFEAF (Montpellier, 8-11 mai 2013), Ausonius, Bordeaux, 669-696.

HEINZ C., \& BARBAZA M., 1998 - Environmental changes during the Late Glacial and Post-Glacial in the central Pyrenees (France): New charcoal analysis and archaeological data. Review of Palaeobotany and Palynology, 104 (1), 1-17.

HEINZ C. \& THIÉBAULT S., 1998 - Characterization and palaeoecological significance of archaeological charcoal assemblages during Late and Post-Glacial phases in southern France. Quaternary Research, 50 (1), 56-68.

HENRY A., 2011 - Paléoenvironnements et gestion des combustibles au Mésolithique dans le sud de la France: anthracologie, ethnoarchéologie et expérimentation. Thèse de doctorat, Université Nice Sophia Antipolis, $297 \mathrm{p}$.

HENRY A., VALDEYRON N., BOUBY L. \& THÉRY-PARISOT I., 2013 - History and evolution of Mesolithic landscapes in the HautQuercy: new charcoal data from archaeological contexts. The Holocene, 23 (1) ,127-136.

KRAUSS-MARGUET I, 1980 -Contribution à l'histoire de la végétation postglaciaire des Grands Causses d'après l'analyse anthracologique du gisement préhistorique de La Poujade (commune de Millau, Aveyron). D.E.S. Montpellier, 79 p.

KRAUSS-MARGUET I., 1981 - Analyse anthracologique du gisement postglaciaire de la Poujade (Millau, Aveyron). Paléobiologie continentale, 12 (1), 1981, 93-110.

IFN, 1995 - Département de l'Aveyron: résultats du troisième inventaire forestier (1994). Nogent-sur-Vernisson, Inventaire Forestier National (IFN), carte en couleur, $30 \mathrm{~cm}, 176 \mathrm{p}$.

IGN [en ligne], 2016 - Géoportail, le portail national de la connaissance du territoire, 2016 [consulté le 01 mai 2017]. Disponible sur https://www.geoportail.gouv.fr/

JACQUIOT C., TRENARD Y. \& DIROL D, 1973 - Atlas d'anatomie des bois des Angiospermes (essences feuillues). Centre Technique du Bois, Paris, $175 \mathrm{p}$.

LEMÉE G., 1953 - Sur la végétation postglaciaire du Lévezou d'après l'analyse pollinique. Bulletin de la Société Botanique de France, 100, 26-29.

LIOU TCHEN-NGO, 1929 - Études sur la géographie botanique des Causses. Extrait des archives de Botanique, III, mémoire n ${ }^{\circ}$, Caen, $220 \mathrm{p}$.

MARRES P., 1936 - Les Grands Causses : étude de géographie physique et humaine. I, Le milieu physique. Imprimerie Arrault, Tours, $208 \mathrm{p}$ 
MARTONNE (de) E., 1909 - Traité de géographie physique. Paris, Armand Colin, $908 \mathrm{p}$.

OLIVIER S., 2012 - Aux marges de l'espace agraire, Inculte et genêt en Lodévois (XVII -XIXe siècle). Thèse de Doctorat, Caen, 2 tomes, $1209 \mathrm{p}$.

PELTIER A., TOUZET M.C., ARMENGAUD C. \& PONGE J.F., 1997 - Establishment of Fagus sylvatica and Fraxinus excelsior in an old-growth beech forest. J. Veg. Sci., 8, 13-20.

PETIT R.J., CSAIKL U.M., BORDACS S., BURG K., COART E., COTTRELL J., VAN DAM B.C., DEANS J.D., DUMOLINLAPEGUE S., FINESCHI S., FINKELDAY R., GILLIES A., GLAZ I., GOICOECHEA P.G., JENSEN J.S., KONIG A., LOWE A.J., MADSEN S.F., MATYAS G., MUNRO R.C., OLALDE M., PEMONGE M.-H., POPESCU F., SLADE D., TABBENER H., TAURCHINI D., DE VRIES S.M.G., ZIEGENHAGEN B. \& KREMER A., 2002 - Chloroplast DNA variation in European white oaks : phylogeography and patterns of diversity based on data from over 2,600 populations. Forest Ecology and Management, 156 (1-3), $5-26$

PRIOTON J., 1932 - Le Causse du Larzac et ses forêts. Revue des Eaux et Forêts, 70 (9), 741-753.

PRIOTON J., 1970 - Flore forestière du Larzac. Causses et Cévennes, 11 (1), 415-422.

PUERTAS O., 1998 - Palynologie dans le delta du Lez : contribution à l'histoire du paysage de Lattes. Lattes, Edition de l'Association pour la recherche archéologique en Languedoc oriental, $181 \mathrm{p}$.

PULIDO AVILA M. G., 2006 - Conséquences de l'anthropisation sur la dynamique postglaciaire de la végétation dans le sud du Massif Central, France. Thèse de doctorat, Université Paul Cézanne, AixMarseille, $308 \mathrm{p}$.

QUEZEL P., BARBERO M., BONNIN G. \& LOISEL R., 1980 Essai de corrélations phytosociologiques et bioclimatiques entre quelques structures actuelles et passées de la végétation méditerranéenne. Naturalia Monspelliensi, 79-87.

RAMEAU J.-C., MANSION D., DUMÉ G. \& GAUBERVILLE C., 2008 - Flore forestière française : guide écologique illustré. 3, Région méditerranéenne. Institut pour le développement forestier, Ministère de 1'Agriculture et de la Pêche, Direction de l'Espace Rural et de la Forêt \& École Nationale du Génie Rural, des Eaux et des Forêts, Paris, 2426 p.

REILLE M., 1990 - Leçons de palynologie et d'analyse pollinique. Éditions du CNRS, Paris, 206 p.

ROIRON P., CHABAL L., FIGUEIRAL I., TERRAL J.-F., \& ALI A., 2013 - Palaeobiogeography of Pinus nigra Arn. subsp. Salzmannii (Dunal) Franco in the north-western Mediterranean Basin, based on macroremains. Review of Palaeobotany and Palynology, 194, 1-11.

SCHWEINGRUBER F.H., 1990 - Anatomie europäischer Hölzerein Atlas zur Bestimmung europäischer Baum-, Strauch- und Zwergstrauchhölzer. Verlag Paul Haupt, Stuttgart, 800 p. [Allemand/ Anglais]

SOLARI, M.-E. \& VERNET, J.-L., 1992 -Late glacial and Holocene vegetation of the Corbières based on charcoal analysis at the Cova de 1'Espérit (Salses, Pyrénées Orientales, France). Review of Palynology and Palaeobotany 71, 111-120.

THERY-PARISOT I., 1998 - Économie des combustibles et paléoécologie en contexte glaciaire et périglaciaire, Paléolithique moyen et supérieur du sud de la France. Anthracologie, expérimentation, taphonomie. Thèse de doctorat, université de Paris-I, $630 \mathrm{p}$.

THERY-PARISOT I., 2001 : Économie des combustibles au Paléolithique, anthracologie, expérimentation, taphonomie. Dossier de Documentation Archéologique ${ }^{\circ}$ 20. CNRS Éditions, 195 p.

THERY-PARISOT I. \& MEIGNEN L., 2000 - Économie des combustibles (bois et lignite) dans l'abri moustérien des Canalettes. Gallia Préhistoire, 42, 45-55.
THÉRY I., GRIL J., VERNET J.-L., MEIGNEN L. \& MAURY J., 1996 - Coal used for Fuel at Two Prehistoric Sites in Southern France: Les Canalettes (Mousterian) and Les Usclades (Mesolithic). Journal of Archaeological Science, 23, 509-512.

THIÉBAUT B., 1982 - Existe-t-il une hêtraie méditerranéenne distincte des autres forêts de hêtre en Europe occidentale? Vegetatio, 50, 23-42.

THIÉBAULT S. \& VERNET, J.-L., 1992 - Végétations méditerranéennes et civilisations préhistoriques : le cas de Font-Juvénal. Les Charbons de bois, les anciens écosystèmes et le rôle de l'Homme, Colloque International, Montpellier, 10-13 septembre 1991. Bulletin de la Société botanique de France, 139 (2-4), 441-450.

TOGNETTI R., JOHNSON J.D. \& MICHELOZZI M., 1997 Ecophysiological responses of Fagus sylvatica seedlings to changing light conditions. I. Interactions between photosynthetic acclimation and photoinhibition during simulated canopy gap formation. Physiologia Plantarum, 101, 115-123

TRIAT-LAVAL H., 1979 - Histoire de la forêt provençale depuis 15000 ans d'après l'analyse pollinique. Forêt Méditerranéenne, 1, 19-24.

VALDEYRON N., BOSC-ZANARDO B. \& BRIAND T., 2008 -Évolutions des armatures de pierre et dynamiques culturelles durant le Mésolithique dans le Sud- Ouest de la France: l'exemple du HautQuercy (Lot, France). In: Petillon JM, Dias-Meirinho MH, Cattelain $\mathrm{P}$ et al. (eds) Recherches sur les armatures de projectiles du Paléolithique supérieur au Néolithique. Actes du colloque C83, XVe congrès de l'UISPP, Lisbonne. Palethnologie, 1, 278-295.

VALDEYRON N., BOSC-ZANARDO B., BRIAND T., HENRY A., MARQUEBIELLE B. \& MICHEL S., 2011 -Le gisement du Cuzoul de Gramat (Lot, France): présentation des nouveaux travaux et résultats préliminaires. In: Senepart I, Perrin T, Thiraut $\mathrm{E}$ et al. (eds) Marges, frontières et transgressions. Actualité de la Recherche. Actes des 8e Rencontres Méridionales de Préhistoire Récente, Marseille, 7-8 nov. 2008. Archives d'écologie préhistorique, Toulouse, p. 197-211.

VALLADAS H., CHADELLE J.-P., GENESTE J.-M., JORON J.-L., MEIGNEN L. \& TEXIER P.-J, 1987 - Datations par la thermoluminescence de gisements moustériens du sud de la France. L'Anthropologie, 91 (1), 211-226.

VANDEN BERGHEN C., 1963 - Note sur la végétation des grands Causses du Massif central de France. Mémoires Société Royale de Belgique, 1, 1-215.

VAQUER J. \& RUAS M.-P., 2009 - La grotte de l'Abeurador, FélinesMinervois (Hérault): Occupations humaines et environnements du Tardiglaciaire à 1'Holocène. De Méditerranée et d'Ailleurs, hommages offerts à J. Guilaine. Archives d'Écologie Préhistorique, Toulouse, 761-792.

VERNET J.-L., 1968 -La couverture forestière des Causses du Massif Central de France au Post-glaciaire, d'après l'étude de charbons de bois préhistoriques. Bulletin de l'Association Française pour l'Étude du Quaternaire, 5 (3), 181-196.

VERNET J.-L., 1972 - Nouvelle contribution à l'histoire de la végétation holocène des Grands Causses d'après les charbons de bois. Bulletin de la Société Botanique de France, 119, sup3, 169-182.

VERNET J.-L., 1973 - Étude sur l'histoire de la végétation du sud-est de la France au Quaternaire d'après les charbons de bois principalement, Paléobiologie Continentale, 4 (1), $90 \mathrm{p}$.

VERNET J.-L., 1985 - Écologie des Causses au Quaternaire. Bulletin de la Société Languedocienne de Géographie, 3-4, 265-286.

VERNET J.-L., OGEREAU P., FIGUEIRAL I., MACHADO YANES C. \& UZQUIANO P., 2001 - Guide d'identification des charbons de bois préhistoriques et récents : Sud-Ouest de l'Europe: France, Péninsule ibérique et îles Canaries. Éditions du CNRS, Paris, $395 \mathrm{p}$. 